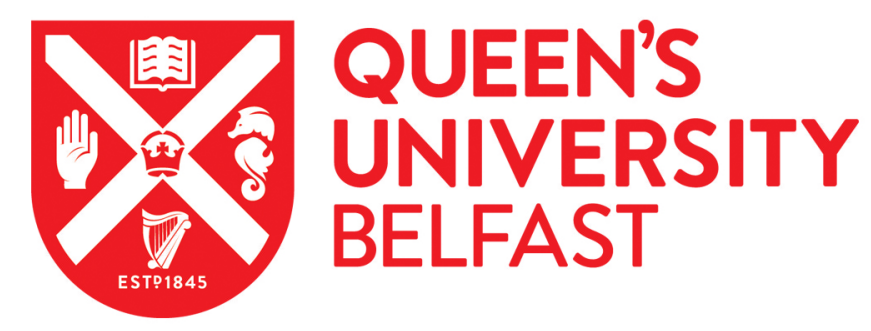

\title{
Low cost bridge load test: calculating bridge displacement from acceleration for load assessment calculations
}

Hester, D., Brownjohn, J., Bocian, M., \& Xu, Y. (2017). Low cost bridge load test: calculating bridge displacement from acceleration for load assessment calculations. Engineering Structures, 143, 358-374. https://doi.org/10.1016/j.engstruct.2017.04.021

\section{Published in:}

Engineering Structures

\section{Document Version:}

Peer reviewed version

\section{Queen's University Belfast - Research Portal:}

Link to publication record in Queen's University Belfast Research Portal

\section{Publisher rights}

(C) 2017 Elsevier Ltd. All rights reserved. This manuscript version is made available under the CC-BY-NC-ND 4.0 license

http://creativecommons.org/licenses/by-nc-nd/4.0/,which permits distribution and reproduction for non-commercial purposes, provided the author and source are cited.

\section{General rights}

Copyright for the publications made accessible via the Queen's University Belfast Research Portal is retained by the author(s) and / or other copyright owners and it is a condition of accessing these publications that users recognise and abide by the legal requirements associated with these rights.

Take down policy

The Research Portal is Queen's institutional repository that provides access to Queen's research output. Every effort has been made to ensure that content in the Research Portal does not infringe any person's rights, or applicable UK laws. If you discover content in the Research Portal that you believe breaches copyright or violates any law, please contact openaccess@qub.ac.uk. 


\title{
Low cost bridge load test: calculating bridge displacement from acceleration for load assessment calculations
}

\author{
David Hester ${ }^{a}$, James Brownjohn ${ }^{b}$, Mateusz Bocianc, Yan Xu' \\ aDepartment of Civil Engineering, Queen's University Belfast, UK \\ bibration Engineering Section, University of Exeter, UK \\ ${ }^{\mathrm{C}}$ Department of Engineering, University of Leicester, UK
}

\begin{abstract}
Bridge failure to pass load capacity assessment is unfortunately not an uncommon problem in bridge engineering and it is a potentially expensive problem for the bridge owner. Using load test data to justify increase in assessed load capacity is recognised as a viable approach in professional codes of practice. However, load tests are rarely carried out in practice because traditionally they are expensive to conduct and may not always justify an increase in assessed load capacity. Therefore this paper proposes a simple, quick and reliable approach for bridge load testing. In particular a procedure to calculate the bridge displacement to a moving truck by double integration of bridge acceleration is presented. Integrating acceleration to calculate displacement is not a new approach, with authors reporting difficulties due to errors in acceleration signals and unknown initial conditions. Many of the previous approaches have focused on developing signal processing algorithms to correct for the signal errors and while some good results have been reported, typically the derived displacements are very sensitive to parameters used in the correction algorithm, such as passband filter frequencies. Consequently, without comparison with directly measured displacement data, reliability of the procedure cannot be established and errors quantified. Therefore in this study a stripped down procedure is applied placing emphasis instead on minimising the errors in the recorded acceleration by using appropriate hardware and developing a quality control procedure that allows the user to assess the likely accuracy of the calculated displacement signal. The effectiveness of the proposed approach is trialled in the laboratory and in the field, with an accuracy of $\pm 0.5 \mathrm{~mm}$ observed.
\end{abstract}

\section{Keywords}

Bridge; Load test; Bridge acceleration; Displacement monitoring;

\subsection{Introduction}

\section{$\underline{1.1 \text { Bridge assessment and load testing }}$}

Load carrying capacity assessment of a bridge generally is carried out using a Finite Element (FE) model of the bridge to calculate the load effects (e.g. bending moments and shear forces) generated due to the prescribed loads. If the load effects predicted by the model are greater than the calculated load capacity of the deck, the bridge owner/operator is faced with a difficult and potentially expensive problem as even limited strengthening works are expensive. The possibility that a given bridge may have reserves of strength in excess of the calculated value (e.g. as shown by field testing in [1]) means that there has been a growing interest in using load test results to justify 
increasing the assessed load capacity following a failed load capacity assessment. Broadly speaking a load test involves placing load(s) of known magnitude on the bridge and measuring displacement and/or strain. Bridge assessment codes (e.g. [2], [3]) recognise the potential benefits of conducting a load test and they specifically allow for their use. Despite this, load tests are rarely carried out for a number of reasons:

(i) traditionally they require specialist equipment and are expensive to implement,

(ii) they slow the decision process because they take significant time to organise and

(iii) there is no guarantee that the test will result in an increase in assessed capacity.

However, in the opinion of the authors, following a failed initial load carrying assessment a quick/reliable method for performing a load test would be very attractive to bridge engineers due to the high potential reward compared to cost. Therefore this paper proposes a simple, reliable, approximate approach for load testing. In particular a procedure to calculate the bridge displacement to a moving load by double integrating bridge acceleration is presented and trialled successfully in the field. The method requires limited equipment (accelerometer, video camera, test truck) and limited planning (no special access equipment or traffic management). Specific details on the proposed method and the results obtained are given in Sections 2-5. However, to give some background on the area, section 1.2 gives a brief overview of conventional sensing systems used for tracking bridge displacement, and Section 1.3 provides an overview of previous work on integrating acceleration signals to calculate displacement. As already mentioned, strain could be measured during a load test but for a quick and easy load testing, strain is not particularly suitable. This is because it is often relatively complicated to access and attach strain gauges to the underside of bridge decks and this motivates the use of easier to deploy accelerometers. (For brevity, the underside of the bridge deck will be hereafter referred to as the 'soffit').

\subsection{Conventional methods for tracking bridge displacement}

Traditionally the difficulty with measuring bridge displacement in the field is the absence of a fixed reference point means that standard displacement measuring sensors such as Linear Variable Differential Transformers (LVDT) cannot be used. A number of bridge displacement monitoring approaches have been implemented, each with their own advantages and disadvantages and the choice of technology for bridge deformation measurements is discussed elsewhere e.g. [4]. Often the method selected depends on factors such as spatial range/resolution, sample rate/frequency response, number of axes and stability of fixed reference points. For short span bridges over accessible land LVDTs can sometimes be used if the bridge deck is not too high and the user is only interested in vertical deflection. For example LVDT's are used in this study to measure the vertical displacement of a bridge and Moreau et al. [5] use LVDT's for a similar purpose However, in reality LVDT's are rarely practicable in the field and not suitable at all for long spans over water and inaccessible open space. For these kind of bridges, options include total stations [6] and hydraulic level sensing [7] however, GPS is increasingly becoming the most common approach. At the end of the 1990s Roberts et al. [8] used GPS to track the displacement of the Humber suspension bridge to a convey of 5 trucks. In more recent work Mochas \& Stiros [9] used GPS to successfully track the displacement of a relatively stiff footbridge. Notwithstanding the work in [9] has limitations, 
particularly for shorter spans where movement ranges are modest. Therefore more recently a number of authors have placed emphasis on trying to identify bridge deflection using vision based systems and some promising results have been reported. Some of these systems are commercially available (e.g. Imetrum [10]) however they tend to be expensive and require a certain level of expertise to operate. Similar vision based systems have been developed by researchers and presented in the literature [11]-[13] and while these have the advantage of being cheaper (than the commercial systems) they take time and expertise to implement. As well as the cost and/or expertise required to use vision based systems, there are challenges to using them in the field, in particular, (i) camera/lens stability and (ii) varying light conditions. In the field the camera will typically be tens of metres from the bridge deck, requiring highly stable camera mounting to avoid significant errors. For example, a camera $30 \mathrm{~m}$ from the bridge deck rotated by just one minute of arc on its support equates to a deck displacement of several millimetres. Typically image tracking algorithms work by tracking a user defined pixel zone of interest (or target) between successive frames, however changing lighting condition might disturb system's capability for target recognition. Finally a number of authors [14]-[19] have looked at the problem of calculating bridge displacement from acceleration and the work of these authors is described in more detail later in this section, however, first a general overview on the topic of calculating displacement from acceleration signals is provided.

\subsection{Integrating acceleration to calculate displacement}

Due to the cost/logistical difficulties associated with the methods mentioned in the previous section this paper explores a simple and low cost approach to calculating approximate displacement from acceleration signals. In particular the challenge of calculating the displacement of a bridge due to the passage of a truck is examined, for a load test without the need for traffic management on bridge.

Aside from not knowing exactly the initial conditions (velocity and displacement), the fundamental problem with integrating acceleration to calculate displacement is amplification of the low frequency noise that inevitably exists in real acceleration data. Thong et al. [20] give a useful overview on the challenge of doubly integrating acceleration measurements containing noise. Essentially measured acceleration and subsequent computation of velocities and displacements are stochastic variables/processes, characterized by errors (noise) and Stirios [21] provides a rigorous mathematical discussion on this issue. He demonstrates how the errors/noise in the acceleration records are amplified and accumulated during the double-integration procedure. More recent work on the same topic by Moschas et al. [22] demonstrates the same thing using experimental data supported by Finite Element Modelling and detailed statistical analysis. The effect of integrating noise is demonstrated on 15 seconds of acceleration data simultaneously recorded by three different accelerometers, P,Q, R placed on a rigid laboratory floor (Fig. 1(a)). The signals in Fig. 1(a) are expected to be almost entirely broadband accelerometer noise with a small component due to genuine sub-micron ground vibrations at frequencies generally above $5 \mathrm{~Hz}$ [23]. The low frequency component of this noise can be exposed by its removal using a moving average filter (a form of low pass filtering).The result after filtering is shown in Fig. 1(b), where the y-axis limits are two orders of magnitude lower than in Fig 1(a). Fig.1(b) shows that signal P has the most low frequency noise, signal $R$ has the least, and signal $Q$ is somewhere in between. The significance of this low frequency noise is evident when acceleration signals P-R shown in Fig. 1(a) are double integrated to displacement, Fig. 1(d). 
Double integrated signals from accelerometer $\mathrm{P}$ suggest that, starting from zero the sensor experienced a maximum displacement of $8 \mathrm{~mm}$ at approximately 6 seconds, thereafter it gradually reduced, reaching $-2 \mathrm{~mm}$ at around 13 seconds where it remained for the rest of the window. Data from accelerometer $Q$ suggest that the sensor experienced an approximately sinusoidal displacement over the course of the 15 seconds with an amplitude of approximately $2 \mathrm{~mm}$. Finally acceleration signals from accelerometer $\mathrm{R}$ suggest a displacement of close to zero for the duration of the 15 second window. If the plot from accelerometer $R$ is examined at a zoomed in scale then displacement in the range $\pm 0.2 \mathrm{~mm}$ are evident.

The double integration from Fig 1(a) to Fig 1(d) involves the following simple steps:

(i) apply a linear de-trend function to the raw acceleration signal (Fig. 1a),

(ii) assume initial velocity is zero, integrate using MATLAB 'cumtrapz' function to calculate velocity (Fig. 1c) and

(iii) assume initial displacement is zero and integrate again to obtain displacement (Fig. 1d).

(a)

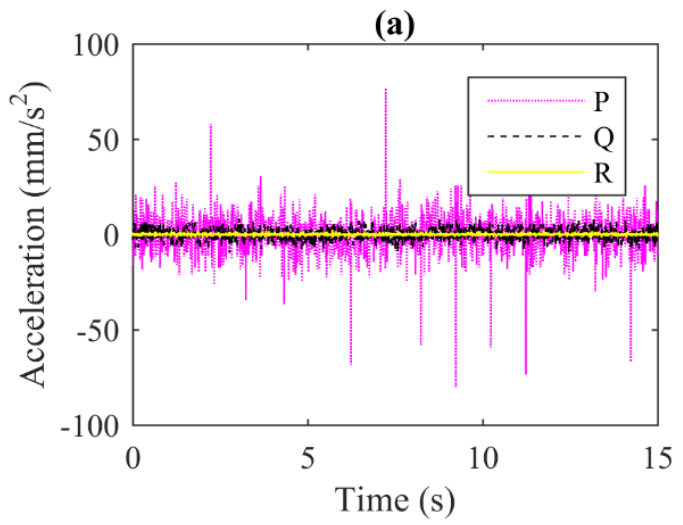

(c)

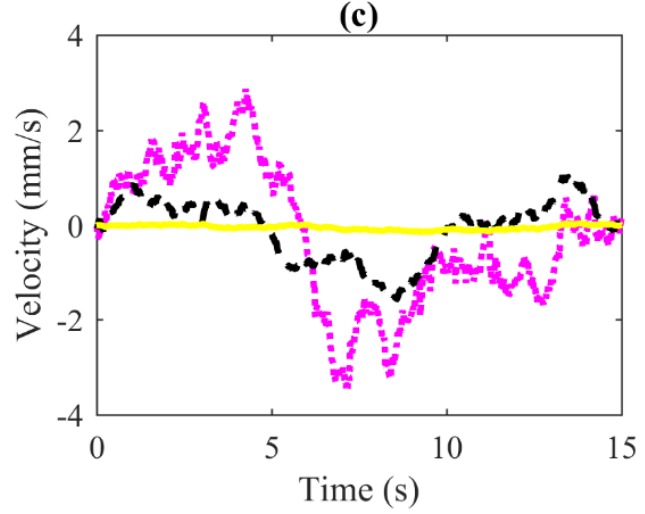

(b)

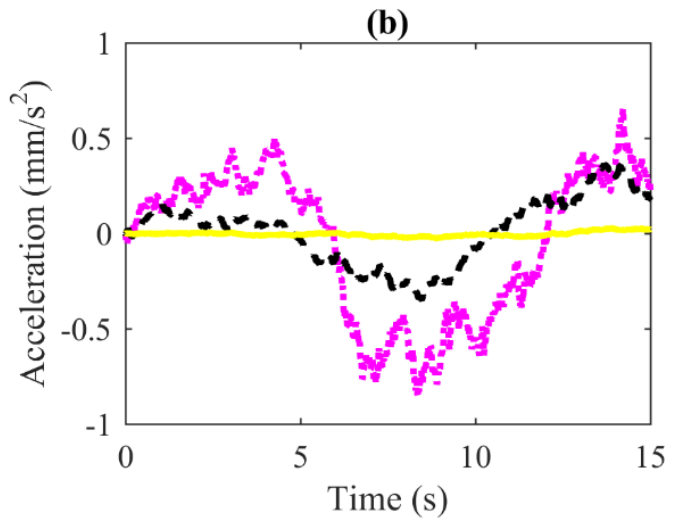

(d)

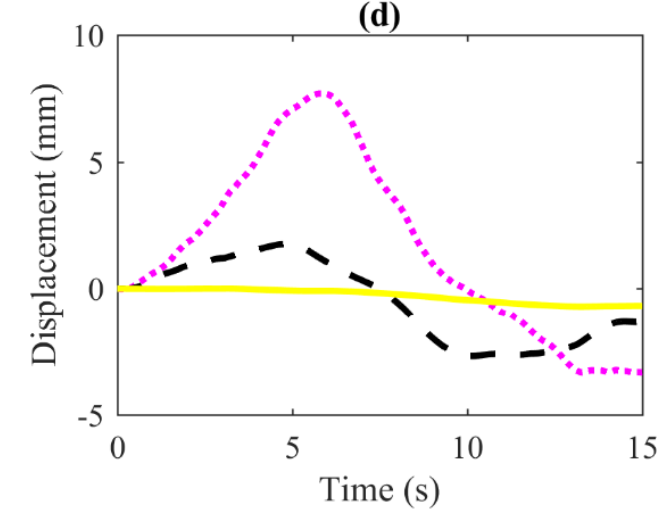

137

Fig. 1, Overview of the challenge of obtaining displacement from acceleration signals, (a) raw acceleration from accelerometers $\mathrm{P}, \mathrm{Q}$ and $\mathrm{R}$ (b) acceleration data after application of moving average filter to expose low frequency component of measurement noise, (c) velocity following $1^{\text {st }}$ integration (sensors with largest low frequency noise in part (b) show largest calculated velocity) (d) displacement following $2^{\text {nd }}$ integration where calculated displacements are approximately proportional to the low frequency noise observed in (b). 
Recovering dynamic displacements of all but the very long span bridges is more straightforward since the spurious displacement demonstrated in Fig. 1 can be partially avoided by high pass filtering the acceleration signal prior to integration. However, to calculate the displacement response of a bridge due to the passage of a truck the low frequency component of the acceleration signal must be retained as this is the part of the spectra that contains quasi-static response associated with the prescribed moving load. In this case it is challenging to identify a suitable high pass filter cut-off frequency.

In the following review of previous research on recovering quasi-static displacements it is observed that the integration procedures involve correction of the signals to conform to an expected shape. A simple example of this is seen in Fig 1. Because the acceleration data were collected from accelerometers placed on rigid ground the true displacement signal in Fig. 1(d) should be a horizontal line of zero displacement. This in turn requires a horizontal line of zero velocity, and to achieve this in the first integration stage a simple 'correction' can be devised. For example, a best fit polynomial fit to signal P in Fig. 1(c) can be subtracted from the signal to leave an approximate flatline velocity which on further integration will yield a more credible displacement. The simple example demonstrates the concept behind all correction methods, i.e. in that the knowledge of the shape of the true displacement signal can be used to establish the approximate shape of the true velocity signal. Then mathematical algorithms can be devised to 'correct' the calculated velocity signal to bring it more in line with the theoretically true velocity signal.

Correction approaches of this type are generally known as a base line correction methods (BCM). Heng et al. [24] give a useful summary of how this approach is implemented, with some of the earliest work in this area directed to recovering permanent ground displacements from accelerograms, or earthquake acceleration records [25], [26]. Other methods use a combination of $\mathrm{BCM}$ and high-pass filtering, e.g. [27]. One of the difficulties with applying BCM to earthquake acceleration records is the possibility that the acceleration record contains a genuine 'fling step', i.e. a true displacement reflecting permanent deformation due to the earthquake. Therefore not something to be corrected for, or removed. However, in structural engineering typically such issues do not arise which makes the use of baseline correction a reasonable approach.

A number of authors have applied BCM related methods to recover truck-induced bridge displacements. Faulkner et al [14] use a combination of BCM and high-pass filtering to calculate the response of a bridge to a moving load, showing that provided a suitable pass-band frequency is chosen, even the forced part of the displacement signal (i.e. the portion of the signal when the truck is on the bridge) can be determined reasonably reliably. However, no guidance is provided on choosing the pass-band frequency for a given bridge which is a major limitation given the strong effect this choice has on the recovered displacement. Paultre et al [15] also use a combination of $\mathrm{BCM}$ and high-pass filtering to calculate the displacement of a bridge due the passage of a truck. Again they show good agreement between calculated displacement and displacement recorded using an LVDT, but no guidance on pass band frequency is provided. Park et al [16] present an excellent study where they propose a correction method they term 'Velocity Estimation Method' (VEM) which shows good performance, but again the issue of parameter choice for the integration method remains problematic. Gindy et al. [17] propose a similar but slightly simpler correction 
method with comparison using an LVDT but in addition they measured bridge velocity experimentally using a laser system to show good agreement at the intermediate stage.

In an effort to calculate the 'approximately true' bridge displacement from an acceleration record, the methods described above attempt to 'correct' the calculated velocity signal to mitigate the natural errors (noise) that occur in the recorded acceleration. Another approach is to 'correct' the recorded acceleration data prior to integration by attempting to fit a mathematical function that captures the true acceleration in the recorded acceleration but not the unwanted noise. For example Kropp [18] showed that an appropriate polynomial fitted to a bridge acceleration signal could be integrated to provide a reasonable indication of the maximum displacement, even if the calculated displacement signal did not exactly follow the displacement signal recorded on the bridge using a deflection gauge. Gindy et al. [19] used a state space model to try to represent the noise free acceleration. Double integration of this modelled acceleration provided a very good match with the displacement signal recorded on the bridge.

The fundamental problem with the approaches described is that the user has to select certain 'parameters' to be used in the algorithm (e.g. the pass band filter frequencies or polynomial order) and which are problematic to choose correctly. Further, if a directly measured displacement signal is available the user can adjust the parameters to obtain the best match. These issues are well demonstrated by Graves [28] who applied correction methods suggested in [29], [30] to acceleration data recorded during a number of different earthquakes, finding that the parameters can be (in his words) 'tuned' until the calculated displacement matched the residual displacement data provided directly by GPS.

It is important to point out that, although Graves [28] was talking about processing earthquake records his observation is equally relevant when dealing with bridge acceleration signals. the ability to find a suitable processing algorithm is significantly increased if the analyst can 'tune' the parameters to match the recorded displacement. However, if these 'tuned' parameters are then applied to acceleration signals from a similar, but slightly different bridge, or to the same bridge with slightly different loading conditions but without directly measured displacement for comparison there is no way to characterise the errors in the calculated displacements

Therefore the focus in this paper is not on developing a new algorithm for calculating bridge displacement from acceleration but rather to:

(i) minimise the errors in the recorded acceleration by using appropriate hardware, and (ii) develop a quality control procedure that allows the user to assess the likely accuracy of the calculated displacement signal.

To this end, Section 2 explains how the static component of the bridge response to be recovered presents in the acceleration signal, Section 3 demonstrates how the accuracy of the calculated displacement is affected by the quality of accelerometer used, Section 4 demonstrates the effectiveness of the proposed approach using data collected during a laboratory trial, and Section 5 validates the proposed approach using data collected in the field. 


\subsection{Basic Theory}

226 This section provides a graphical overview of how the static component of a bridge displacement

227 signal presents in the corresponding acceleration signal. To demonstrate this a simple model, 228 developed in MATLAB and described in detail in [31], simulates a point load crossing a beam. Figs.

229 2(a)-(c) show the mid-span displacement, velocity and acceleration, respectively, as a point load

230 travelling at $4 \mathrm{~m} / \mathrm{s}$ crosses a beam with a span of $20 \mathrm{~m}$. This is a very crude representation but

231 modelling the truck as a single point force is sufficient to demonstrate in Fig. 2(a) the combination of

232 static and dynamic bridge response to a moving truck. The load does not enter the bridge until 2.5

233 seconds into the simulation and equally 2.5 seconds of post load signal is included at the end of the

234 simulation. The 'pre-load' and 'post-load' indicated in Fig. 2(a) are included to replicate the kind of

235 signals that will be integrated later in the paper, i.e. a short portion before the truck arrives, the

236 forced part where the truck is on the bridge, and a short part of the post-load signal after the truck

237 leaves. Fig. 2(d) shows the static and dynamic components of the displacement signal shown in (a) as

238 dashed and solid plots, respectively. The static component is obtained by incrementally moving the

239 load across the bridge, applying it statically at each location and calculating the mid-span

240 displacement. The dynamic component is calculated by subtracting the static component from the

241 signal shown in Fig 2(a). Fig. 2(e) shows the component parts of the velocity signal, obtained by

242 differentiating the static and dynamic components of displacement shown in (d). Finally Fig. 2(f)

243 shows the static and dynamic components of the acceleration signal calculated by differentiating the

244 velocity components in (e). In Fig. 2(f) it can been seen that the amplitude of the dynamic

245 component of acceleration totally dwarfs the static component which appears as an almost straight

246 line. However, Fig. 3(a) reproduces the dashed curve of Fig. 2(f) at a magnified scale revealing it to

247 have a triangular shape. Fig. 3(b) shows the frequency content of the signal shown in Fig. 3(a) with

248 the expected energy concentration at the low end of the spectrum. In fact it is the static component

249 shown in Fig. 3(a) that when integrated will provide the static component of displacement. The

250 difficulty is acceleration signals recorded on real structures will inevitably contain some level of

251 unwanted low frequency noise, distorting the static component of acceleration, resulting in errors in

252 the calculated displacement. In the next section the error (in $\mathrm{mm}$ ) that can be expected from five

253 different accelerometers is quantified. 
(a)

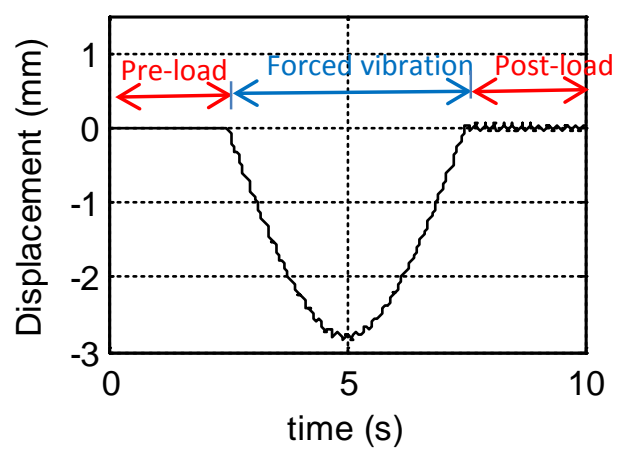

(b)

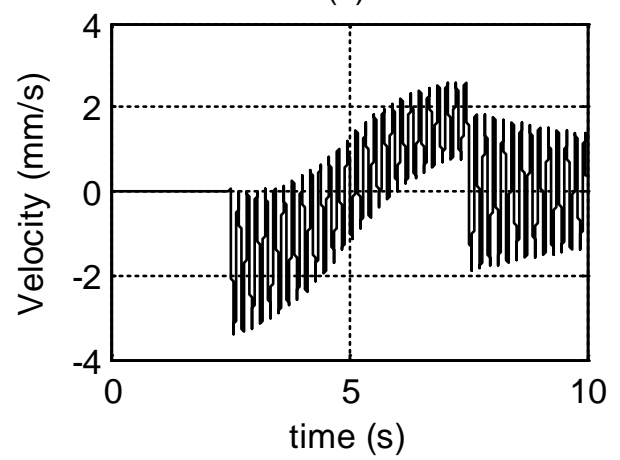

(c)

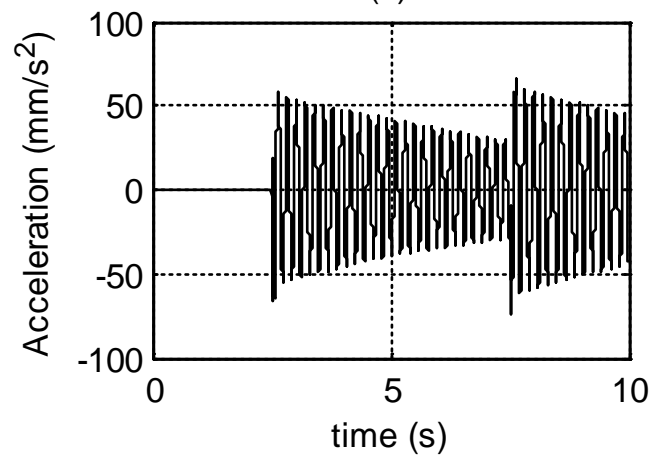

(d)

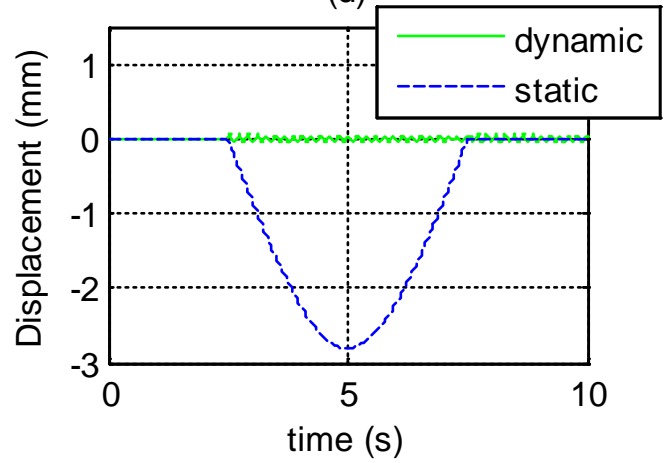

(e)

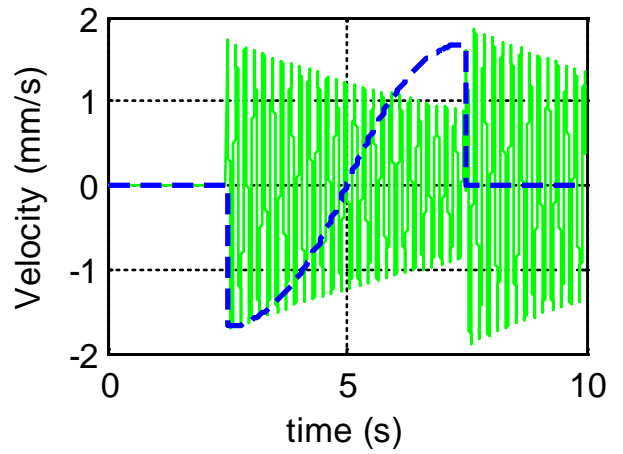

(f)

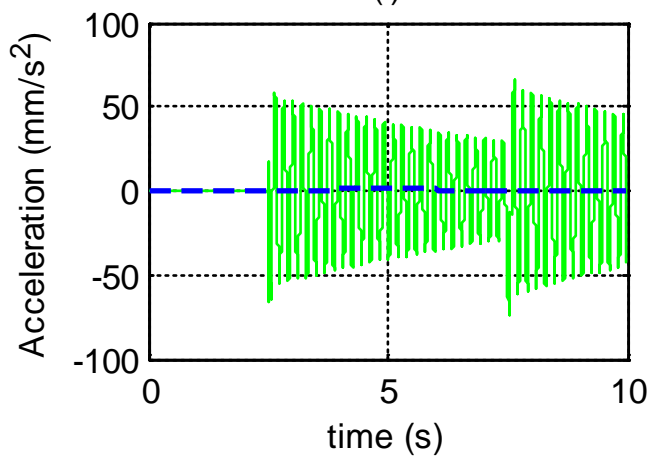

255 Fig. 2, Total response of the mid-span section and its components due to a force travelling at $4 \mathrm{~m} / \mathrm{s}$ :

256 (a) total displacement; (b) total velocity; (c) total acceleration; (d) components of displacement; (e) 257 components of velocity; (f) components of acceleration. 
(a)

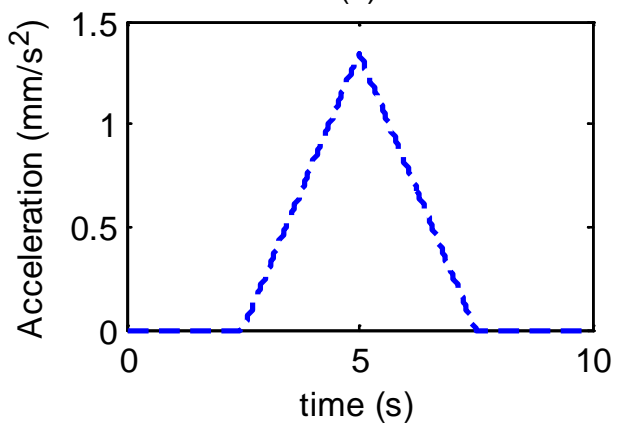

(b)

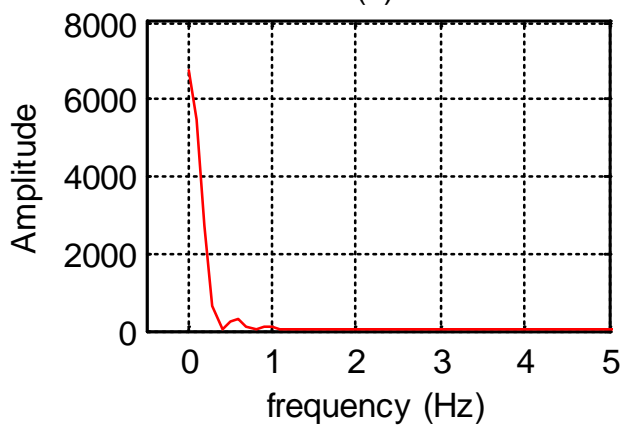

Fig. 3, Static components of acceleration signal (a) in time domain (b) in frequency domain

\subsection{Trialling Different Accelerometers}

As already explained when choosing the accelerometer the most important consideration is that the low frequency noise is minimised because it will dominate errors in calculated static displacement. Therefore the performance of a number of different accelerometers is examined by calculating displacements from acceleration signals recorded on a (rigid) floor in the laboratory. Knowing the correct results to be 'zero' allows an assessment of the errors to be expected when using each type of accelerometer. Some types of accelerometer are inherently unsuited for recovering quasi-static displacements and are not used in this experiment, for example piezoelectric accelerometers designed for shock and vibration applications usually cannot reliably capture frequencies below 0.5 $\mathrm{Hz}$.

In this study five different accelerometers are trialled, four micro-electrical-mechanical-system (MEMS) accelerometers (GCDC, Opal, K-Beam, JA) and one force balance accelerometer (QA), all capable of detecting static acceleration due to gravity, i.e. at $0 \mathrm{~Hz}$. Fig. 4 shows these five accelerometers, and a brief description of each is given below.

GCDC: Manufactured by Gulf Coast Data Concepts (Fig. 4(a)), it is robust, inexpensive and very easy to use/deploy. Using a simple .txt file the user specifies parameters such as scanning rate, acceleration threshold above which data is to be recorded, etc., and the data can be downloaded via the USB connection. The specification sheet for the sensor does not specify the noise that can be expected. The accelerometer in the device is a triaxial MEMS Kionix KXRB5-2050 and while the 'noise density' for this accelerometer is available in the literature $\left(0.00044 \mathrm{~m} / \mathrm{s}^{2} / \mathrm{vHz}\right)$, this will be less than the total sensor noise.

Opal: Manufactured by APMD Inc, these are designed for use as part of a wireless sensor network in biomechanical applications (Fig. 4(b)). The device contains a gyroscopes and magnetometers as well as a triaxial MEMS accelerometer. The specification states accelerometer noise to be 0.0012 $\mathrm{m} / \mathrm{s}^{2} / \mathrm{VHz}$.

K-Beam: Manufactured by Kistler, the type 8315A (Fig. 4(c)) is a uniaxial MEMS accelerometer with a measurement range of $\pm 2 \mathrm{~g}$. The specification states accelerometer noise to be $0.00025 \mathrm{~m} / \mathrm{s}^{2} / \mathrm{vHz}$. 


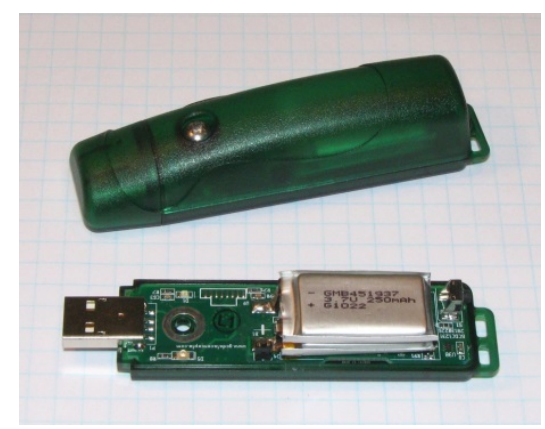

(a)

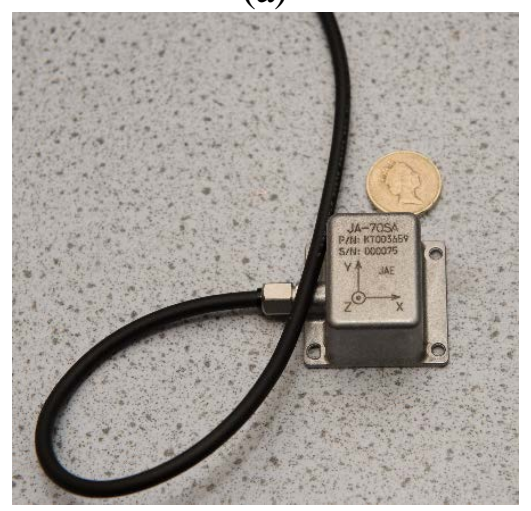

(d) ground.

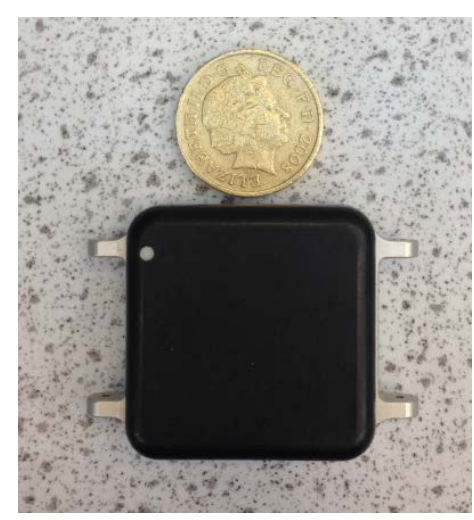

(b)

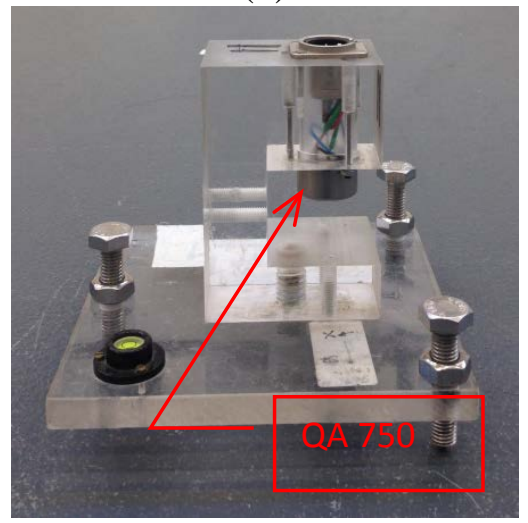

(e)

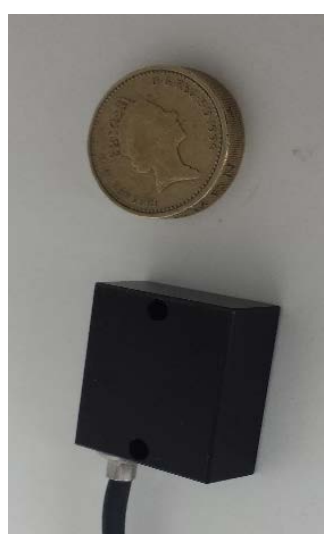

(c)

JA: The JA-70SA manufactured by Japan Aviation Electronics is an extremely low noise MEMS triaxial

QA: The navigation grade force balance accelerometer (QA-750) manufactured by Honeywell (Fig. $4(\mathrm{e}))$ is uniaxial sensor and the most expensive device in this trial. However, they provide excellent performance with respect to noise, $<0.000069 \mathrm{~m} / \mathrm{s}^{2} / \mathrm{VHz}(0-10 \mathrm{~Hz})$ and $<0.00069 \mathrm{~m} / \mathrm{s}^{2} / \mathrm{VHz}(10-500$ $\mathrm{Hz}$ ). Fig. 4(e) shows a photo of the QA accelerometer mounted in a perspex housing with cable connectors and a perspex base plate with three steel foot screws for levelling when used on uneven

Fig.4, Accelerometers trialled in this study (a) GCDC: low cost MEMS accelerometer with on board power and logging capability, (b) Opal: higher quality MEMS accelerometer with on board power and logging capability , (c) K-Beam: conventional MEMS accelerometer which requires external power and data logger , (d) JA high quality MEMS accelerometer which requires external power and data logger, (e) QA: Aviation grade force balance accelerometer which requires external power and data logger .

120 seconds of acceleration signal from the GCDC, Opal, K-Beam, JA and QA accelerometers placed on the rigid laboratory floor are shown in Figs. 5(a) to (e), respectively. Each signal was recorded with a scanning frequency of $128 \mathrm{~Hz}$. The $y$-axis limits in each of the five plots (a-e) are the same and 
309 it is immediately obvious that the GCDC has the largest noise, the QA and JA accelerometers have 310 the least noise, with the level of noise in the Opal and K-Beam accelerometers somewhere in 311 between. This is consistent with the noise levels in the sensor specifications. The noise evident in 312 plots (a)-(e) is high frequency noise, although as shown in section 2 it is actually the low frequency 313 part of the acceleration that most strongly affects calculating displacement from acceleration. To 314 get a feeling for what this low frequency noise looks like in the time domain a 6-second moving 315 average filter was applied to each of the signals shown in Fig. 5 (a)-(e) to remove the high frequency 316 component with the result shown in Fig 5(f) using y axis limits 20 times smaller than those used in 317 plots (a-e). Again the GCDC exhibits the most noise, the QA and JA contain the least noise, with the 318 Opal and the K-Beam somewhere in between. The moving average filter played no further role in 319 this study. 

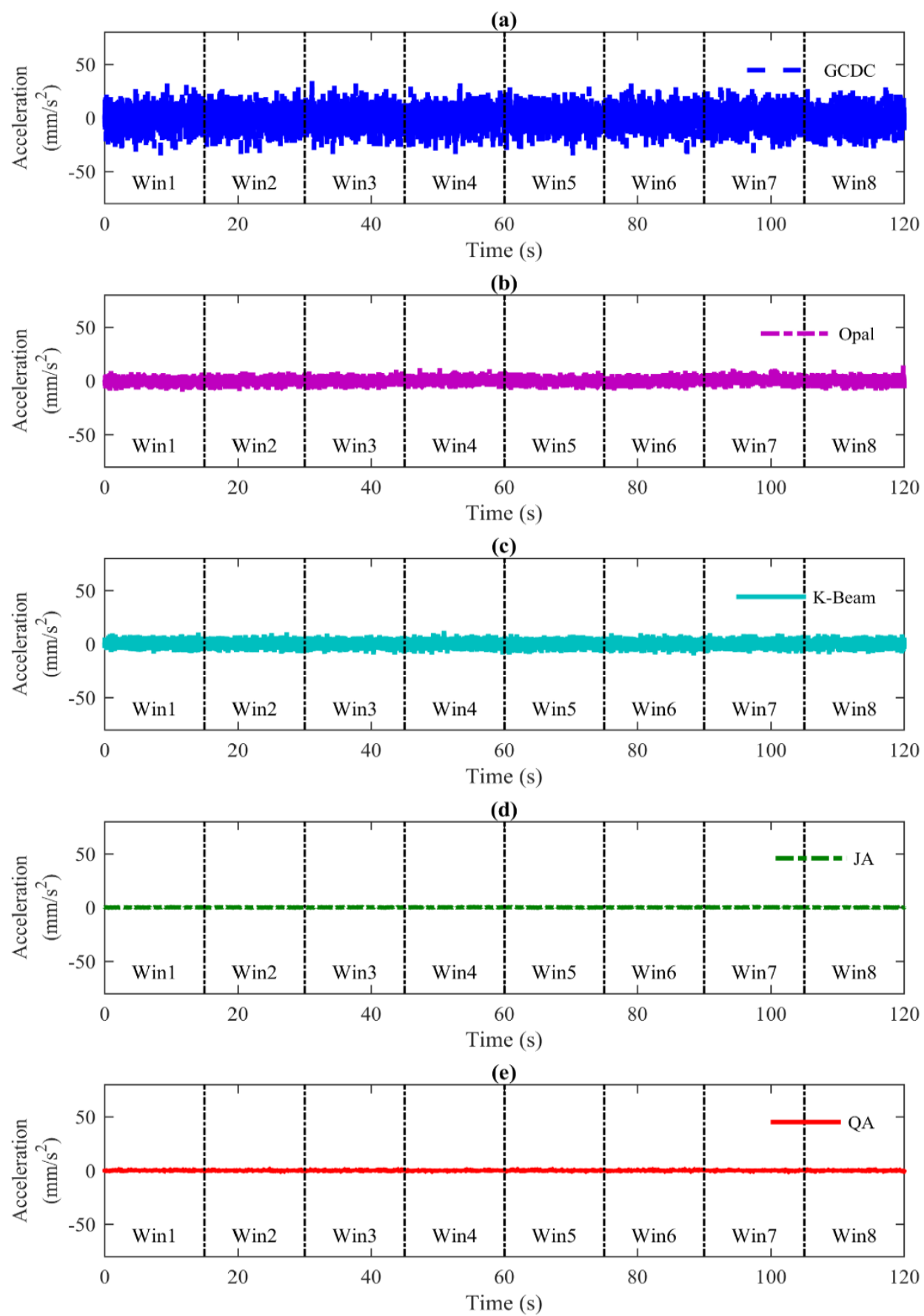

(f)

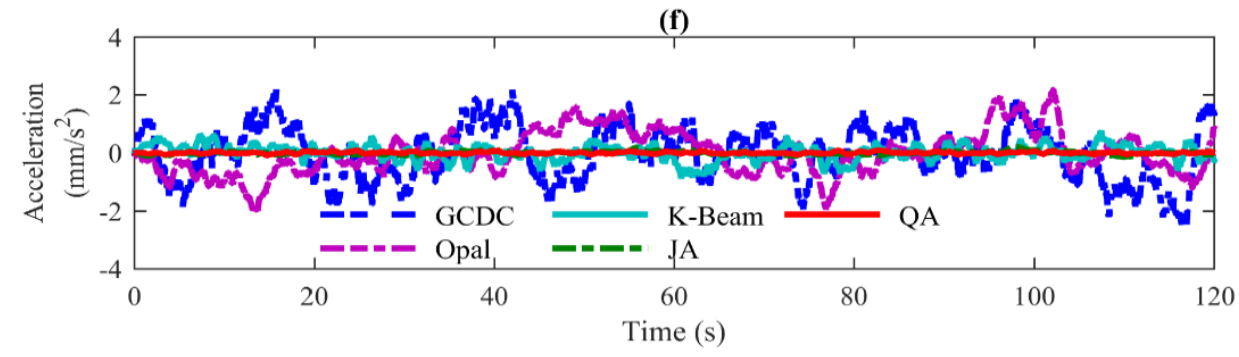

321 Fig. 5, Acceleration signals recorded from five different accelerometers placed on the rigid

322 laboratory floor (a) GCDC accelerometer, (b) Opal accelerometer, (c) K-Beam accelerometer, (d) JA 323 accelerometer, (e) QA accelerometer, (f) signals from parts (a-e) of the figure after smoothing with a moving average filter. 
In Fig. 5(a-e), eight separate 15-second partitions/windows (Win 1-Win 8) are included on the figure to indicate that the signals are not integrated as whole 120 -second signals but as a sequence of short independent signals, to illustrate the range of errors that can be expected when integrating field acceleration signals to displacement. 15 seconds in duration is chosen as it is a realistic duration for a typical highway bridge including ambient signal before the trucks arrival, plus the forced portion of the signal (when the truck is on the bridge) and the short period of ambient vibration immediately after the truck leaves (see Fig. 2(a)).

Fig. 6 shows the result of double integrating the signals in windows 1-4 of Fig 5. In particular Fig. 6(a) shows the result of double integrating the first 15 seconds of acceleration recorded by each of the five sensors, i.e. the 'Win1' data from Figs 5(a)-(e). In Fig. 6(a), the dashed (GCDC) plot shows the result of double integrating window 1 (Win1) of the 'GCDC' signal shown in Fig. 5(a). Similarly the other plots in Fig 6(a) are the result of double integrating the window 1 data from parts (b), (c), (d) and (e) of Fig. 5. All the plots in Fig. 6(a) are calculated assuming zero initial displacement and velocity. Signals are de-trended using the MATLAB linear de-trend function then double integrated using the MATLAB 'cumtrapz' function. No other filtering or correction procedures are applied.

In Fig. 6(a) GCDC data indicate a maximum displacement of $10 \mathrm{~mm}$ at approximately 5 seconds. Also in Fig. 6(a) the window 1 acceleration data from the other sensors produce a displacements at least an order of magnitude smaller. A similar pattern is observed for window 2 acceleration data in Fig. $6(b)$, i.e. all sensors except the GCDC predict displacements close to zero (at the displacement limits adopted in the plot). However, window 3 acceleration data (Fig. 6(c)) indicate a maximum displacement of $6 \mathrm{~mm}$ at approximately 38 seconds for the Opal.
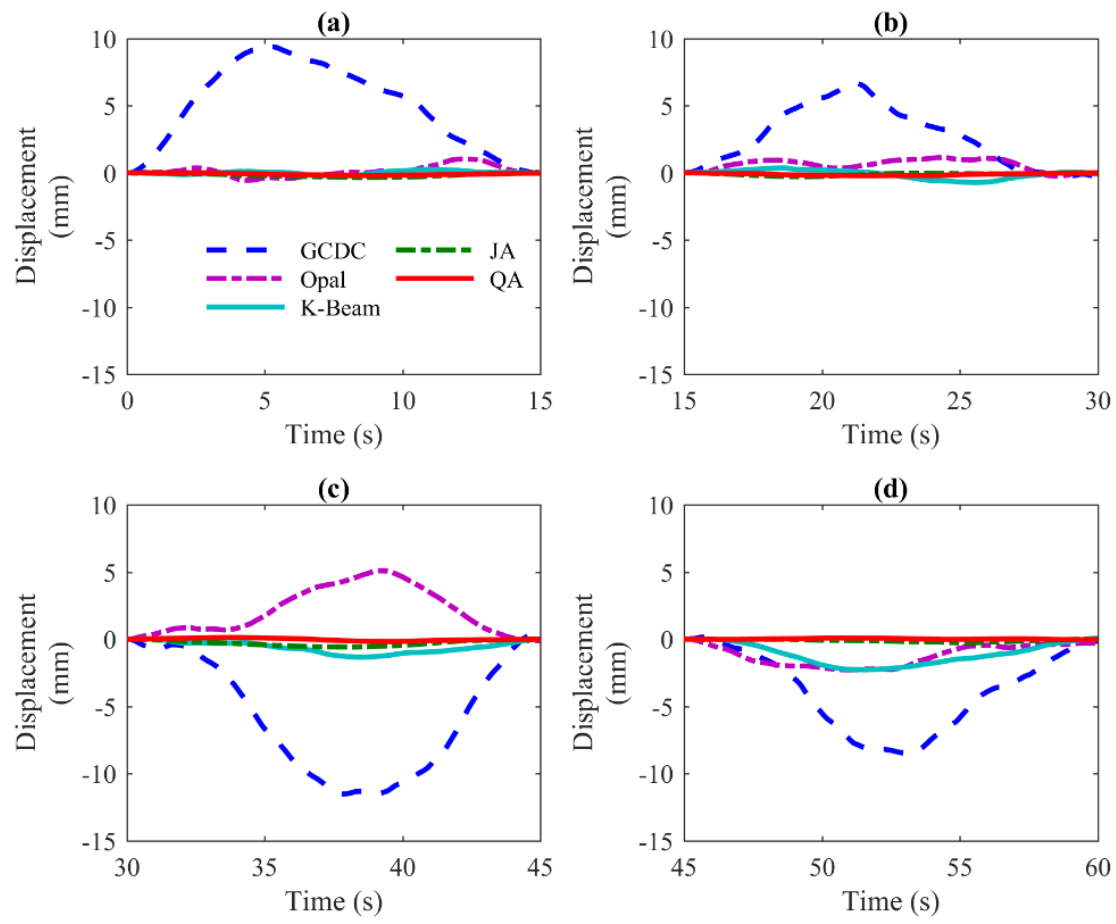

Fig. 6, Displacements calcualted following the double integration of the acceleration signals shown in Fig. 5; (a) Window 1 data, (b) Window 2 data, (c) Window 3 data, (d) Window 4 data. 
350 Fig. 6 tells us that GCDC and Opal accelerometers should not be used for this application but is not informative about errors in the displacement calculated using the other three accelerometers, i.e. KBeam, JA and QA. Therefore a zoomed in view of the displacement calculated from these sensors is shown in Fig. 7 showing the errors in the displacement calculated from the K-Beam accelerometer to be larger than those from either the JA or QA accelerometers. JA and QA performance are comparable, with the QA appearing to perform slightly better, hence the QA was selected for the laboratory and field trials.
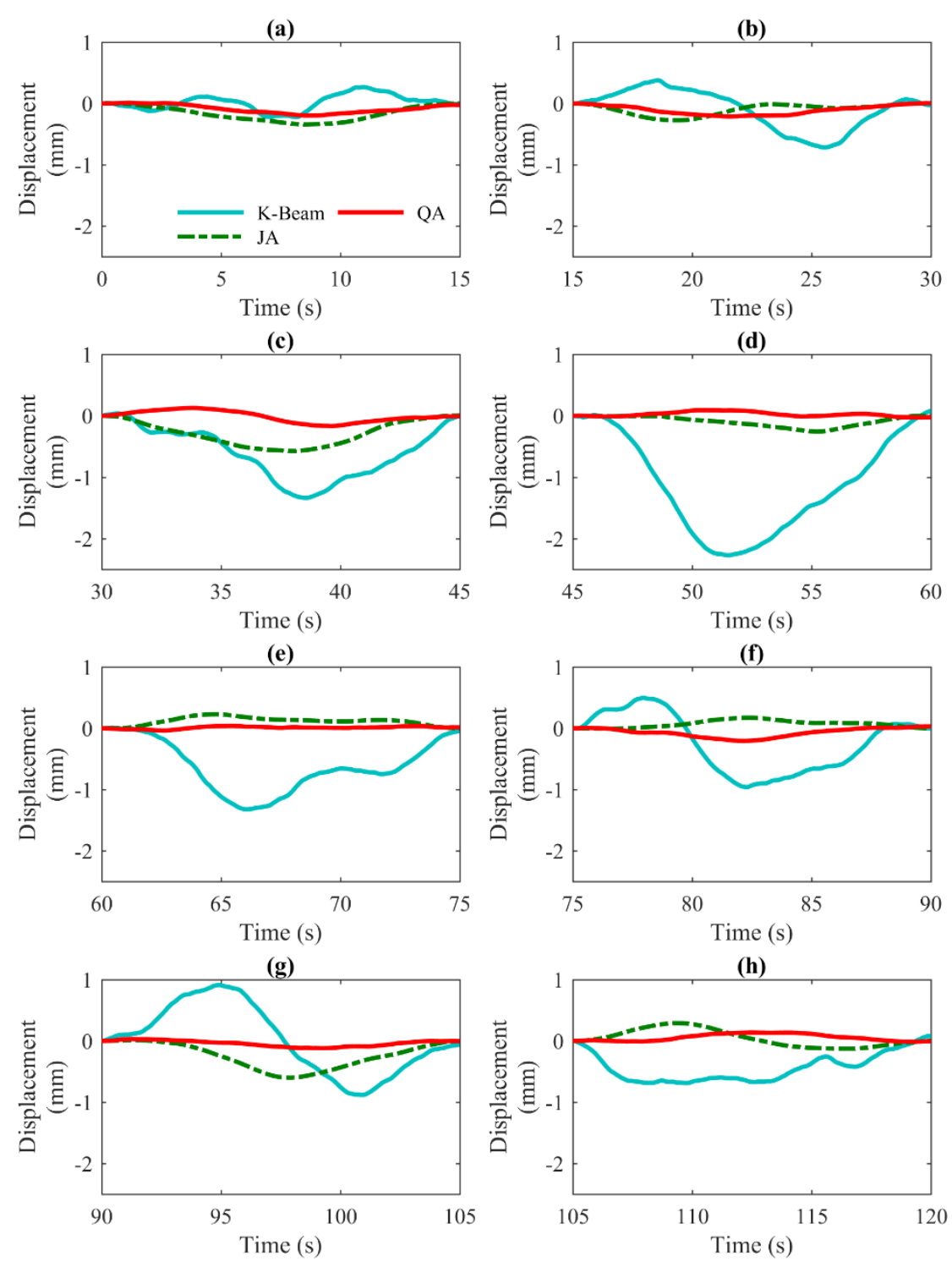

Fig. 7, Displacements calcualted following the double integration of the acceleration signals shown in Fig 5; (a) Window 1 data, (b) Window 2 data, (c) Window 3 data, (d) Window 4 data, (e) Window 5 data, (f) Window 6 data, (g) Window 7 data, (h) Window 8 data 
362 Fig. 8 shows only the QA displacements, with movements in the region of $\pm 0.2 \mathrm{~mm}$ observed. For the field application the bridge quasi-static deflection due to truck crossing is expected to be around 3 $\mathrm{mm}$ (based on hand calculations using bridge properties and approximate truck weight) so errors in the region of $\pm 0.2 \mathrm{~mm}$ are acceptable.

(a)

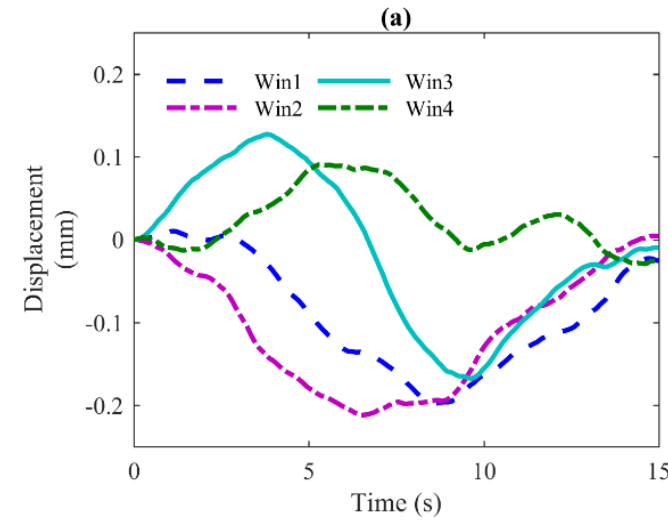

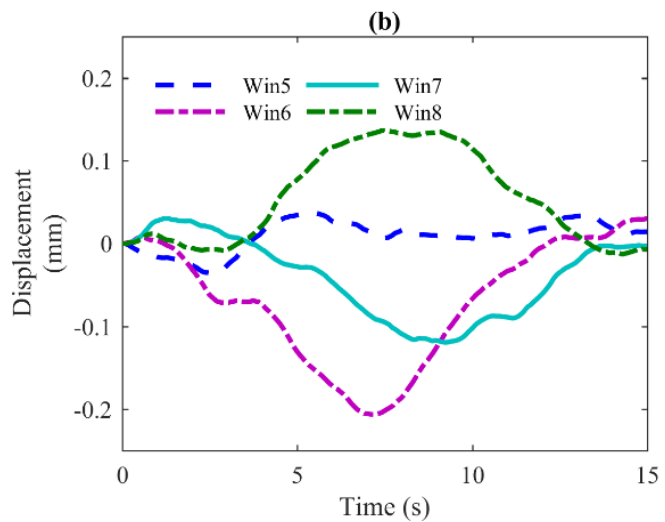

366

367

368

369

370

371

372

373

374

375

Fig. 8, Magnified views of QA displacements shown in Fig. 7, (a) QA displacements calcualted for windows 1-4, (b) QA displacements calcualted for windows 5-8.

\subsection{Laboratory trial}

As the goal of this paper is to examine the feasibility and accuracy of calculating bridge displacement by analysing the bridge acceleration response to the passage of a moving load a directly measured displacement record is required, in this case provided by a conventional LVDT and an optics-based motion capture system (MCS). Before deploying in a field test the accuracy of the test procedure was verified in a laboratory trial. Sections 4.1 and 4.2 below respectively describe the test setup used and the results obtained and the field test is described in section 5 .

\subsection{Test setup}

For maximum realism an analog bridge (i.e. the laboratory test structure) was set up that would have both natural frequency and displacement magnitude similar to what was expected on the real bridge, estimated as $4.9 \mathrm{~Hz}$ and 3-4mm, respectively based on simple calculations and information from available design drawings. Once set up the analog bridge had a natural frequency of $4.7 \mathrm{~Hz}$ and the displacement to the static load was $3.2 \mathrm{~mm}$.

The analog bridge (Fig. 9) comprised a sheet of laminated chipboard spanning between two supports representing the bridge 'deck', and the moving load was a small metal cylindrical weight pulled smoothly along the bridge using a string. In Fig. 9 the cylindrical weight is on the right end of the bridge, a QA accelerometer with an optical target of concentric circles is placed at mid-span on the analog bridge, and the insert (top left) shows an LVDT mounted on a retort stand touching the bridge soffit.

The camera used in the MCS is mounted on the on the smaller surveying tripod in Fig. 9 is zoomed in on the optical target. The camera on the taller tripod having a wide angle lens was used not for tracking but to record video of the experiment. However, the cameras are synchronised so that 
displacement response from the MCS could be interpreted using the video footage. The camera system used is a commercial system manufactured by Imetrum [10].

The reason for using two (direct) displacement measurement techniques (i.e. the LVDT and the MCS) was to check the capability of the MCS, which is a logistically superior system for site testing, as in many situations it is impractical to mount an LVDT under a bridge soffit. Once the three measurement systems were set up the test was carried out by pulling the cylindrical weight across the bridge. The results of the test are presented in the next section.

As well as experiencing acceleration due to the vertical translation of the deck as the load moves across, the accelerometer will also rotate as the deck deforms. Using the same simulation presented in Fig. 2 with deflection $(3 \mathrm{~mm}$ ), in line with what is expected to be encountered in the field test, the maximum rotation is 0.000339 degrees. The resulting cosine error is negligible $\left(\cos \left(0.000339^{\circ}\right) \approx 1\right)$, hence can be ignored.

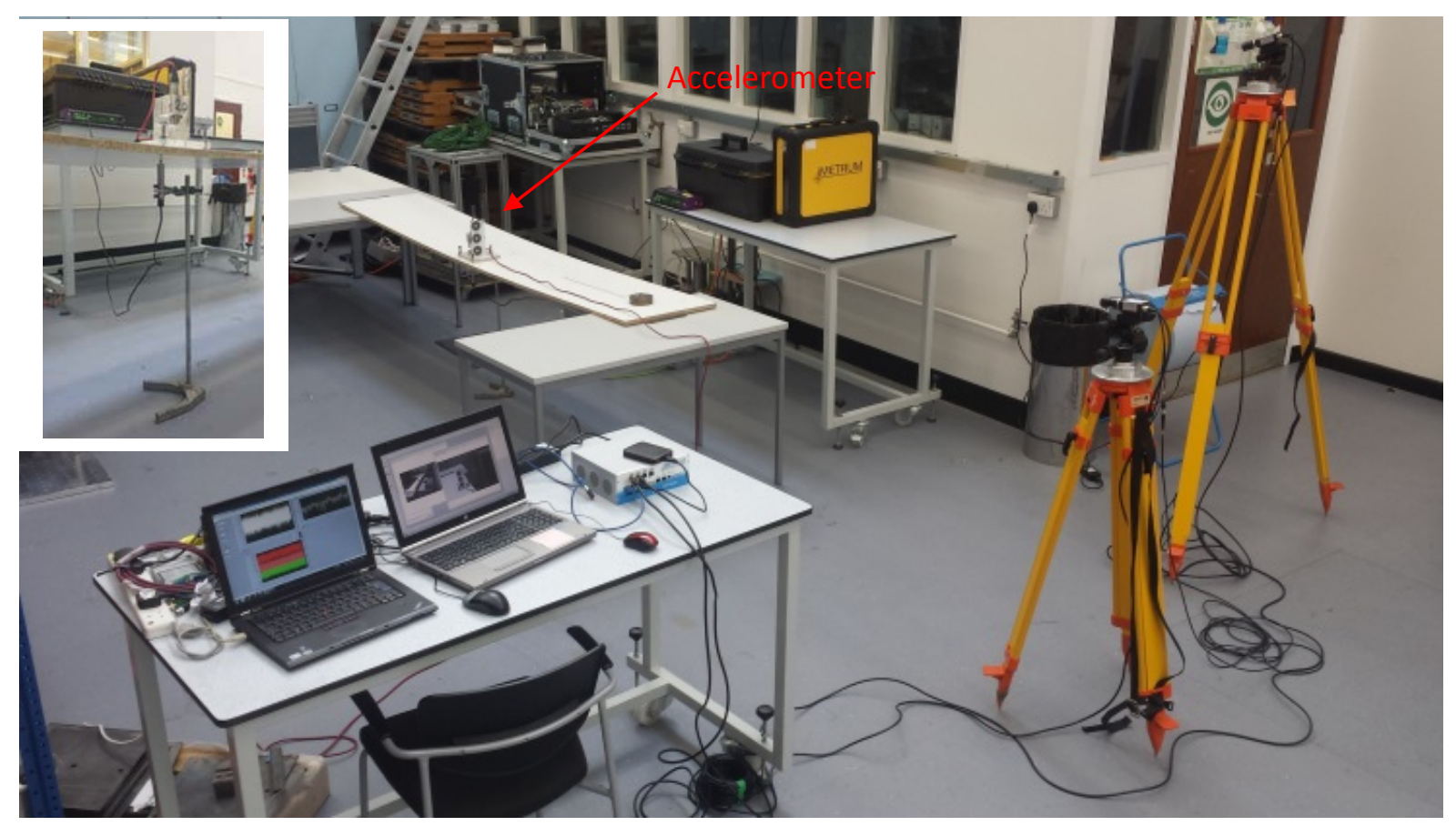

Fig. 9, Experimental setup used in the laboratory trial including 3 measurement systems: (i) Imetrum camera system, (ii) QA accelerometer and (iii)LVDT.

\subsection{Results}

Fig. 10 shows the acceleration measured by the QA accelerometer and the displacement measured by both the Imetrum camera system and the LVDT. In total six separate passes of the moving weight were carried out and this took almost 250 seconds. In the figure acceleration y-axis is on the left hand side and displacement is the $y$-axis on the right hand side. The raw acceleration (topmost curve) is measured in units of gravity ( $\mathrm{g}$ ) with the $1 \mathrm{~g}$ average because the sensor is sitting upright. Six separate pulses of acceleration corresponding to the six separate passes of the moving mass can be seen. The peak displacement is approximately $3 \mathrm{~mm}$ and there is very good agreement between 
415

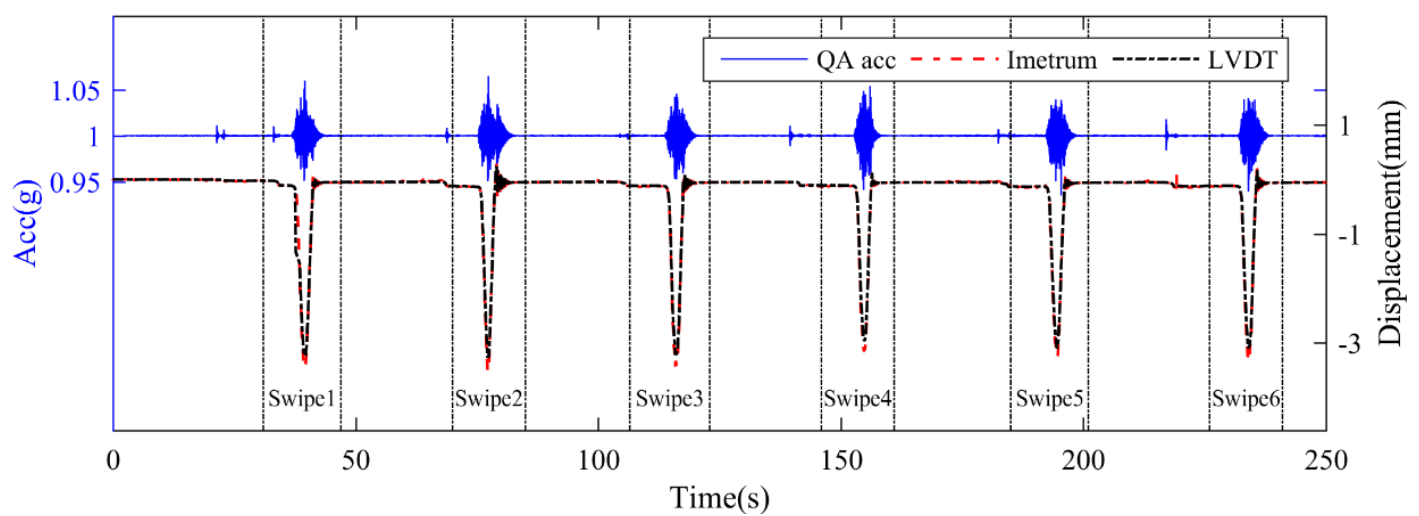

Fig. 10, Raw data from the three sensing systems for the 6 passes of the load, acceleration data (QA) is plotted with respect to the $y$-axis on the left and corresponding data from the two displacement measuring systems (Imetrum \& LVDT) are plotted with respect to the $y$-axis on the right.

To test the reliability of calculating displacement from acceleration the acceleration data are cut into 6 separate 'swipes' (1-6) each approximately 15 seconds long, as indicated in Fig. 10 and centred on the approximate centre of the respective acceleration pulse. (In the context of this paper 'swipe' implies a discreet region of interest relating to the passage of the load which is cut from a larger signal).

Once the 'swipe' acceleration data is extracted it is de-trended and scaled to units of $\mathrm{mm} / \mathrm{s}^{2}$, with examples for swipe 1 and swipe 2 shown in Figs. 11(a) and (d) respectively. Similar to the previous examples, integration takes bridge initial displacement and velocity as zero. The mid-span velocity for swipe 1 is obtained by applying the MATLAB 'cumtrapz' function to the acceleration signal in Fig. 11(a) and the ressult is shown in Fig. 11(b). The integration procedure is repeated on the signal shown in Fig. 11(b) with the result shown in Fig. 11(c). Figs 11(d), (e) and (f) show the recorded acceleration, calculated velocity and calculated displacement, respectively, for swipe 2 .

The method assumes that displacement is zero before the arrival of the truck ('pre load', see Fig. 2) and returns to zero once the truck leaves the bridge ('post-load', see Fig. 2). This may not be true for a real bridge since e.g. locking of the bearings would result in a residual displacement, but it is a reasonable assumption. The video camera recording the experiment allows the reliability of the integration to be checked as follows. From the video camera it is known that the load arrived on the analog bridge at 38 seconds and left at approximately 41 seconds (the entry and exit times are indicated as vertical dashed and solid lines respectively in Fig. 11(c)), hence the calculated displacement before arrival (dashed vertical line) and after departure (solid vertical line) should be approximately zero. However, Fig. 11(c) shows calculated displacement as less than zero from about 35 seconds (reaching approximately $-0.25 \mathrm{~mm}$ just before arrival), not returning to zero as the truck leaves (approx. $-0.1 \mathrm{~mm}$ ) and gradually increasing to become positive (approx. $0.1 \mathrm{~mm}$ ).

The fact that the pre-load and post-load parts of the calculated displacement signal have small errors means that the displacement calculated for the loaded portion of the signal is also likely to have some small errors. The video of swipe 2 shows the load going onto the analog bridge at approximately 76 seconds and exiting at 78 seconds, as indicated on Fig 11(f) using dashed and solid 
lines, respectively. Similar to part (c) of the figure, in Fig. 11(e) the calculated displacement before 76 seconds, and after 78 seconds is close to zero $( \pm 0.1 \mathrm{~mm})$, i.e. the calculated displacement for the unloaded parts of the signal prove relatively accurate so it is likely that the peak displacement in the loaded portion of the signal is also reasonably accurate. Examination of swipes 3-6 show pre-load and post-load displacements to be mostly in the range $\pm 0.15 \mathrm{~mm}$ so over all six swipes the errors are 451 less than $0.3 \mathrm{~mm}$.

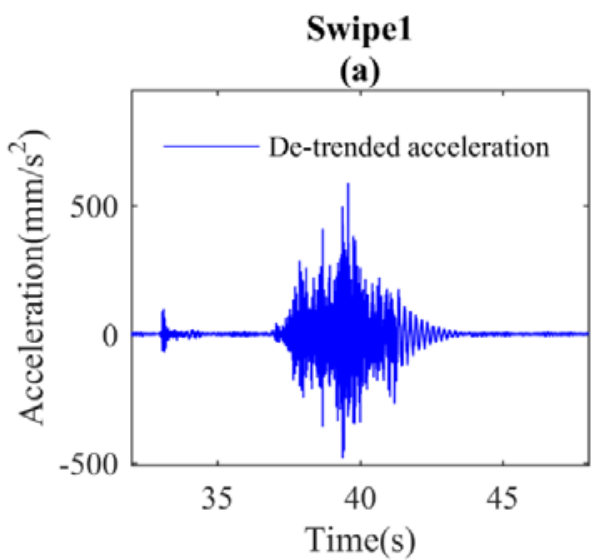

(b)

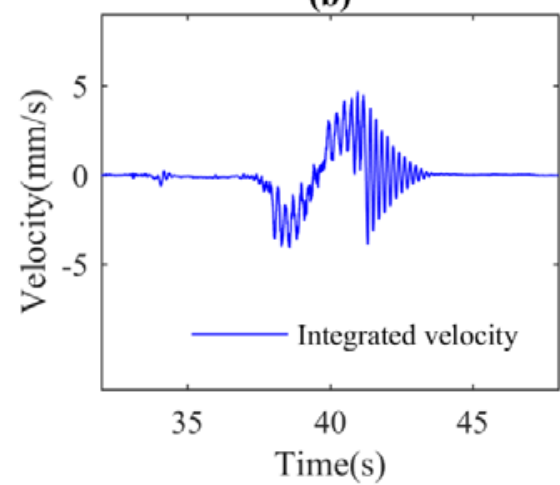

(c)

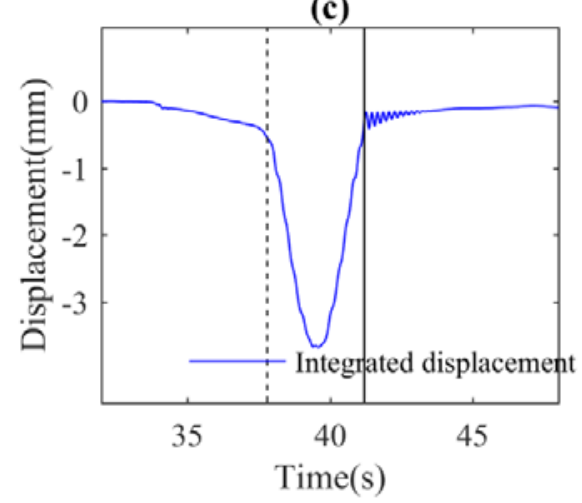

Swipe2

(d)

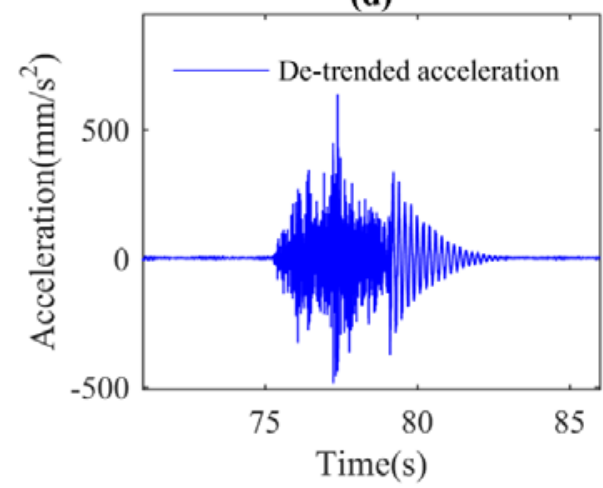

(e)
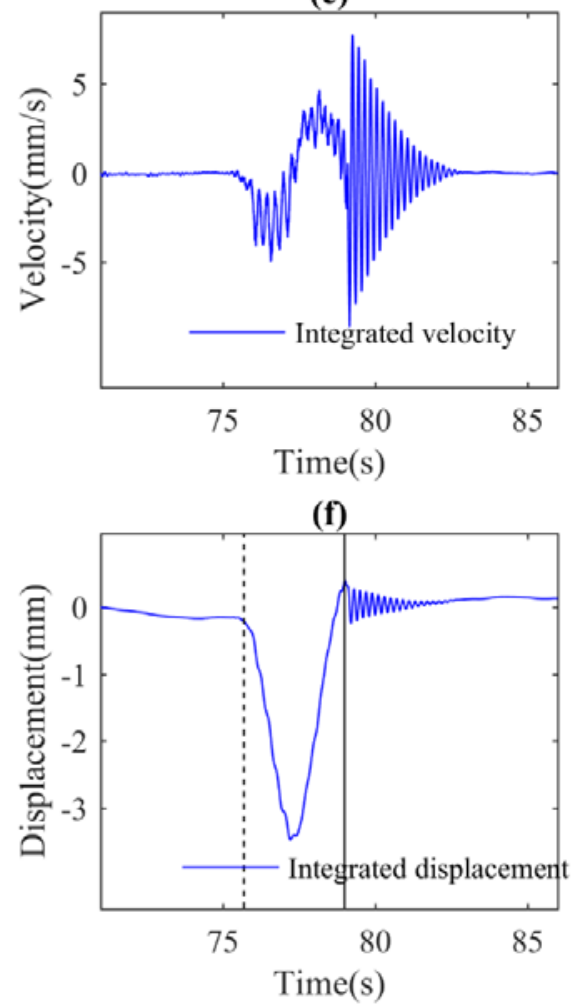

453 Fig. 11, Calculating displacement from acceleration for swipe 1 (left column) and swipe 2 (right column) of laboratory test, (a) Swipe 1 acceleration, (b) swipe 1 velocity calculated following integration, (c) swipe 1 displacement calculated following integration, (d) Swipe 2 acceleration, (e) swipe 2 velocity calculated following integration, (f) swipe 2 displacement calculated following integration. 

also includes the displacement measured directly using the LVDT and Imetrum in order to check the suggested errors. Fig. 12(c)-(f) shows the same thing for swipes 3-6 respectively. It can be seen in Fig. 12 that the calculated displacements for the loaded portion of the signals are very close to the directly measured displacements, which is in line with expectations based on errors observed in the pre-load and post-load portions of the signal. However, some errors are evident, for example in swipe 1 (Fig. 12(c)) the calculated displacement overestimates the peak displacement by about 0.3 $\mathrm{mm}$. This level of error is consistent with the magnitude of the error that was pointed out in the preloaded portion of swipe 1 (see Fig. 11(c)). Similarly in swipe 4 the calculated peak displacement is approximately $0.2 \mathrm{~mm}$ larger than the directly measured value, but this level of error in the peak displacement is consistent with the error at the start of the post-load portion of swipe 4 . The pattern for swipes 2, 3, 5 and 6 (Figs. 12(b), (c), (e) and (f), respectively) is similar, i.e. the errors in the calculated displacement (from the correct zero value) in the pre-load and post-load parts of the signal are representative of errors likely to be present in the loaded portion of the signal.

In effect it appears that the pre-load and post-load parts of the calculated displacement signal can be used as a quality indicator for the accuracy of the loaded part of the calculated displacement signal. This is an important result as it provides an estimation of accuracy in the absence of directly measured displacements.

Comparing displacements calculated through the double integration of acceleration records to displacements measured using some direct means has been reported by other authors. For example Psimoulis et al [32] have compared the displacement calculated following the double-integration of earthquake acceleration records with corresponding ground movements derived from GPS records. In their study they observed that the consistency of the calculated displacement depended on the direction of motion. If the accelerometer used in the study had been a tri-axial accelerometer it would have been interesting to calculate the displacement in the lateral and longitudinal directions as it is possible that calculated movements in these directions could used as quality indicators. That is to say movements in these direction should be very small relative to the vertical movement.

In the same way that in the analog bridge test many swipes were used to investigate the error it is sensible to do a number of truck passes in a field test. During these experiments it was found that the magnitude of the errors in the pre-load and post-load parts of the calculated displacement signal can be affected by the start time of the swipe. The amplitude of the static peak tends not to be effected by the swipe start time however, to minimise the errors in the pre load and post load sections of the calculated signal it is prudent to try a few closely spaced start times.

492 Although the errors observed in Fig 12 could probably be corrected by using some of the methods discussed in Section 1 the aim here is to avoid the problems of choosing the correction parameters, and instead develop a robust procedure with inbuilt quality control check. The next section describes field application of the proposed procedure. 
(a) Swipe 1

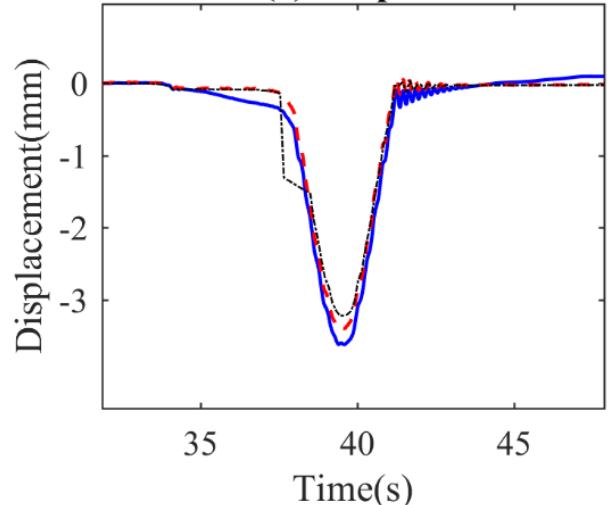

(c) Swipe 3

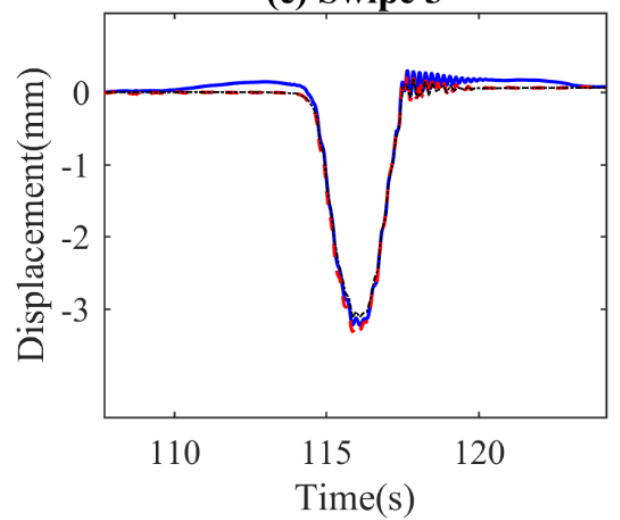

(e) Swipe 5

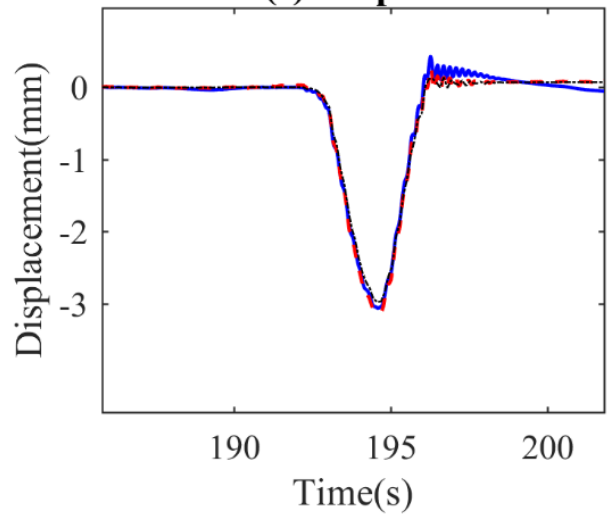

(b) Swipe 2

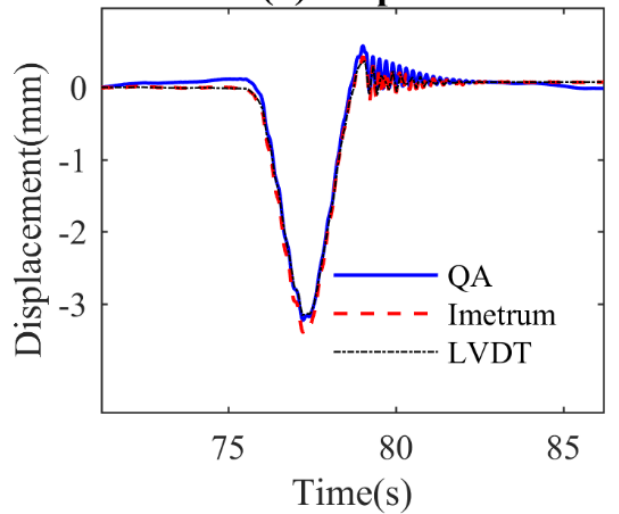

(d) Swipe 4

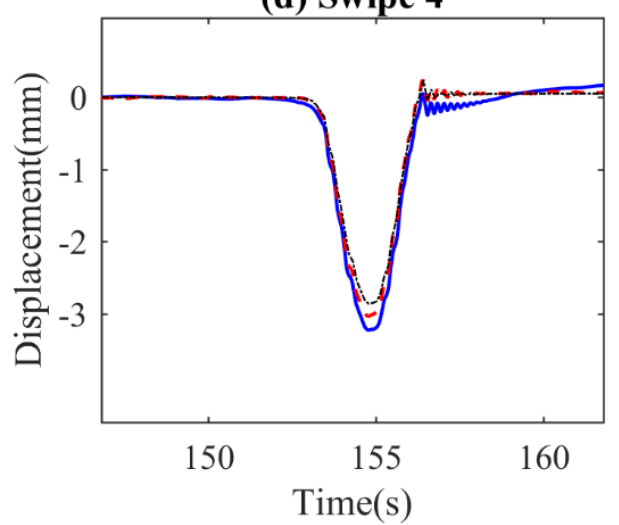

(f) Swipe 6

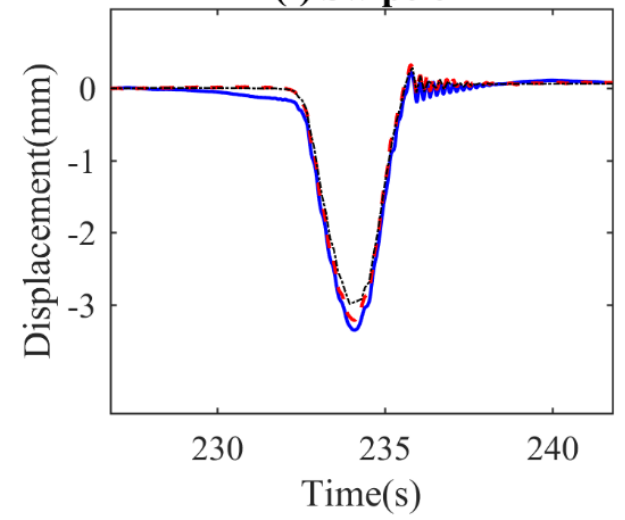

497

Fig. 12, Displacement observed for the three measurement systems for swipes 1-6 (a) swipe 1, (b) swipe 2, (c) swipe 3, (d) swipe 4, (e) swipe 5, (f) swipe 6. 
Following the laboratory trials, the proposed approach was applied on a real bridge.

505

506

507

508

509

510

511

512

513

514

515

516

517

518

519

520

521

522

523

524

525

526

527

528

529

530

531

532

533

534

535

536

537

538

539

540

\subsection{Test Bridge}

The three span concrete road bridge shown Fig. 13(a) used for the field application has beam and slab deck with three separate spans simply supported on piers. The cross section in Fig. 13(b) shows $19 \mathrm{~m}$ span steel I-sections encased in concrete, at $1.54 \mathrm{~m}$ centres.

To install the LVDT a bridge with a relatively low soffit and safe access was required. Fortunately, to facilitate river works, the level of the river had been reduced at the time of the measurement, exposing a sandbank below the middle of the span nearest to the footpath that could be used as a working platform. Details of the expected natural frequency and deflection are given in section 4.1.

\subsection{Test set up}

Fig. 13(c) shows the bridge in plan indicating footpaths on the east and west side of the bridge and four northbound traffic lanes in between. The road layout in the vicinity of the bridge is such that to get multiple passes of the truck in a relatively short time the truck needed to turn right immediately on exiting the bridge. In order to do this safely it needed to travel in lane 4, so it was decided to monitor the beam that was approximately in the middle of lane 4 , which happened to be the third beam from the east side of the bridge. The beam and the monitoring location are indicated in Fig.13(c), while the step ladder visible under the northern span in Fig. 13(a) shows the approximate location where deflection was monitored. The sensing equipment installed was the same as was used in the laboratory test, i.e. an accelerometer, an LVDT and the Imetrum camera system. Further details on installation are given below.

Fig. 13(d) shows a view of the deck soffit with the instrumentation attached at the mid-span and Fig. 13(e) zooms in on the instrumentation. A steel angle is clamped to the corner of the beam allowing the accelerometer to be attached using a magnet. As in the laboratory test an Imetrum optical target was stuck to the side of the accelerometer. The web of the concrete-encased beam could have made an ideal natural target but the view was blocked by the soffit panels.

The final part of the sensing system to be installed was the LVDT. A small dimple was bored in the steel angle to provide a seating for the tip of the LVDT, which was mounted on the top of a telescopic aluminium pole visible to the left of the ladder in Fig. 13(d). Provided the telescopic pole is installed approximately plumb, the spring loading on the tip of the LVDT should suffice to keep the pole/LVDT stable during a test. However, winds speeds on the day of the test exceeded $30 \mathrm{mph}$ resulting in some movement of the pole and leading to some small inaccuracies in the LVDT data. These are indicated in the next section but they did not prove significant.

The wind also impacted location of the camera which was positioned to avoid wind-buffeting and consequent measurement errors. The wind was from the west so camera 1 which was tracking the target at the mid-span of beam 3 was positioned as close to the lee side of the north abutment as line of sight would allow. Camera 2, for recording bridge traffic during the measurements was positioned further south for a wide field of view to be able to see the whole deck and could sustain 


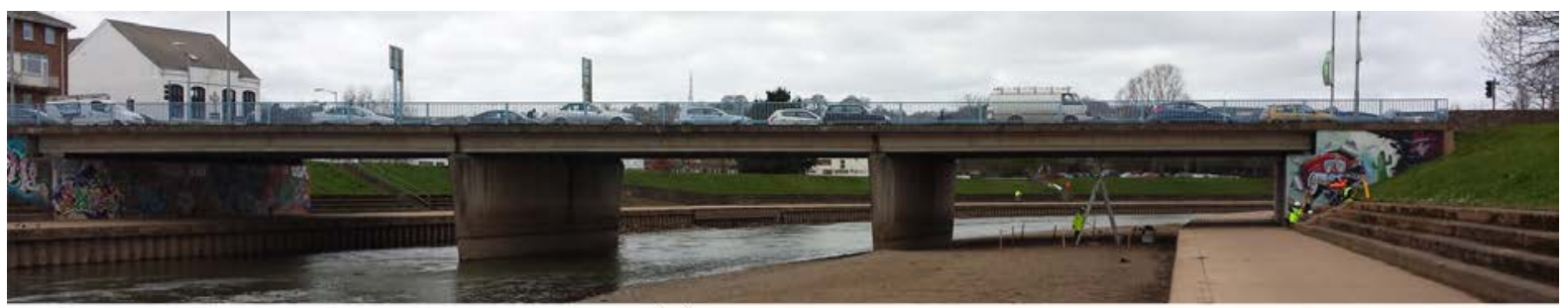

(a)

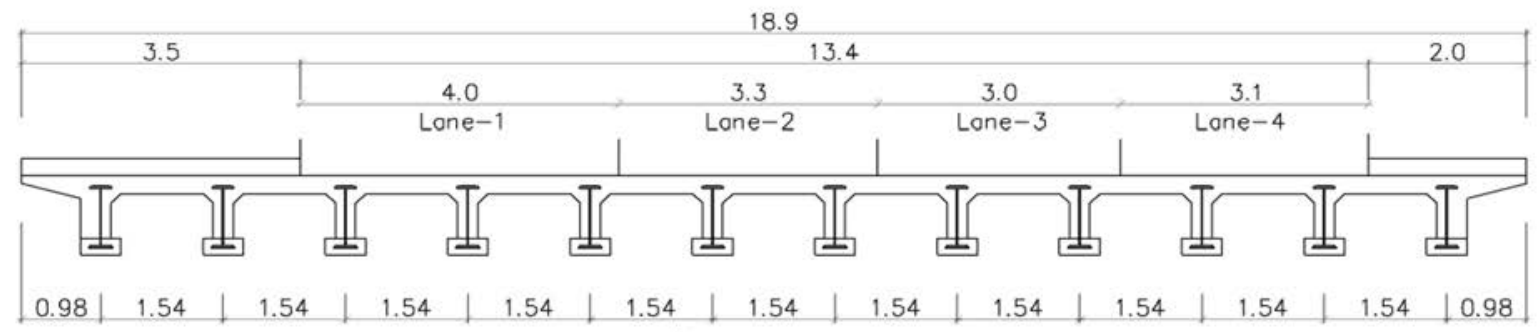

(b)

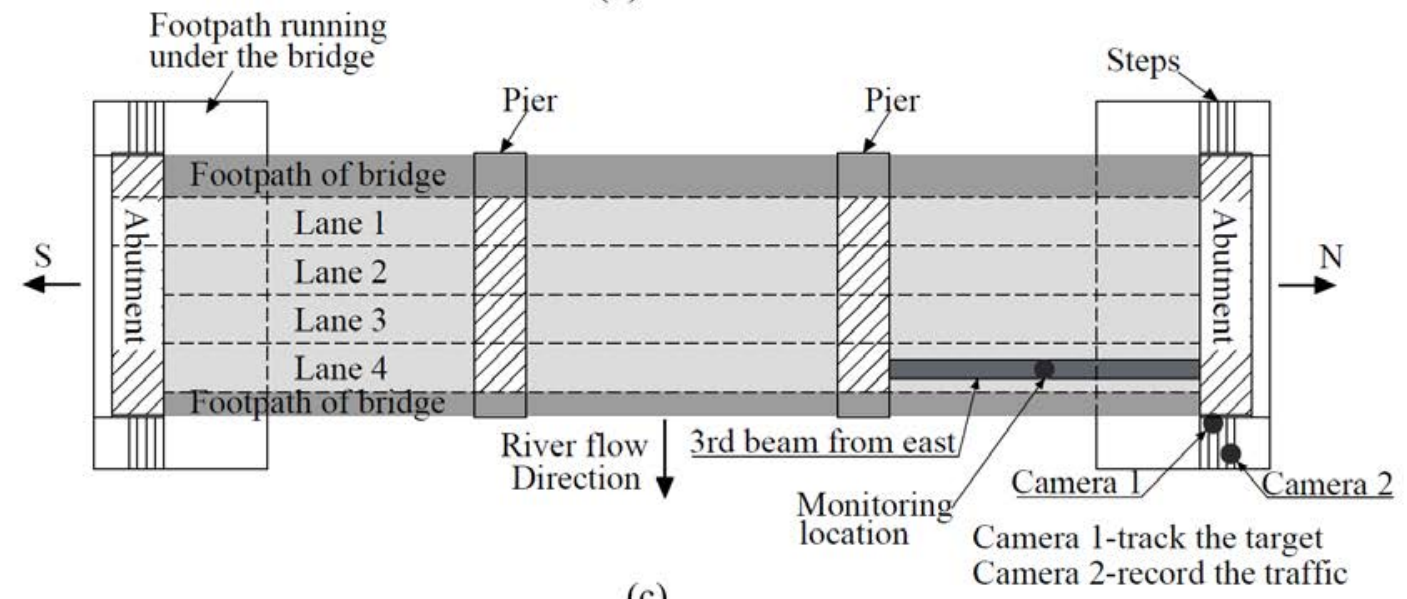

(c)

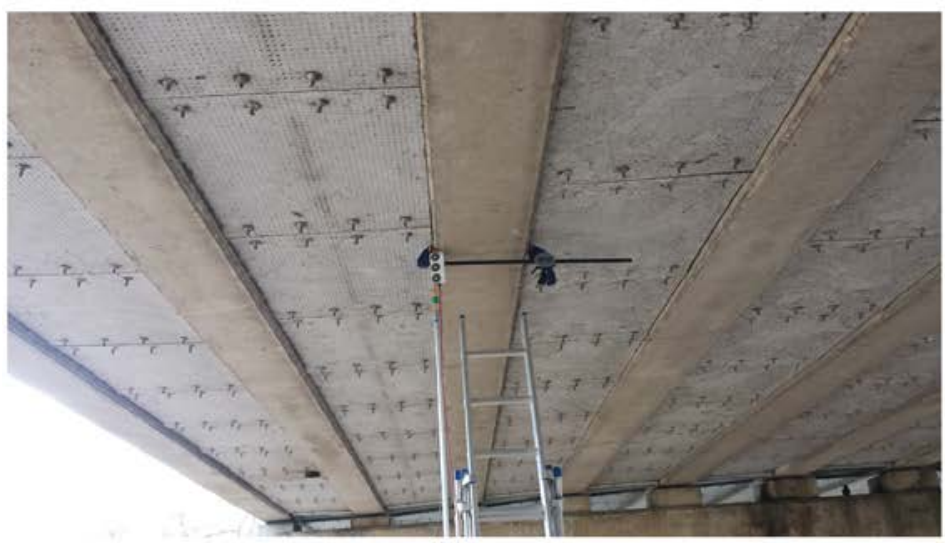

(d)

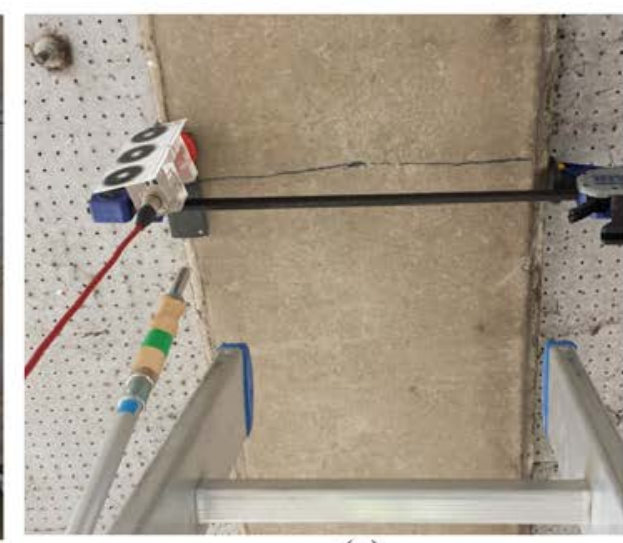

(e)

Fig. 13, Bridge used in field test, (a)side view of the bridge from the East, (b) Typical vertical cross section through the deck (c) Schematic of test setup, (d) view of deck soffit with instrumentation installed, (e) Zoomed in view of instrumentation attached to soffit of the beam, accelerometer installed but LVDT not yet in place 
5.3 Vehicle used

551 The truck used in the test was the four axle Scania P410 shown in Fig. 14(a); part (b) of the figure 552 shows the axle spacing of the truck. On the morning of the test the truck was loaded with stone 553 aggregate and its gross weight was 32 tonnes. The approximate weight on each axle is indicated in 554 Fig. 14(b) and it is assumed that the load on a given axle is equally distributed to between the wheels 555 on the axle.

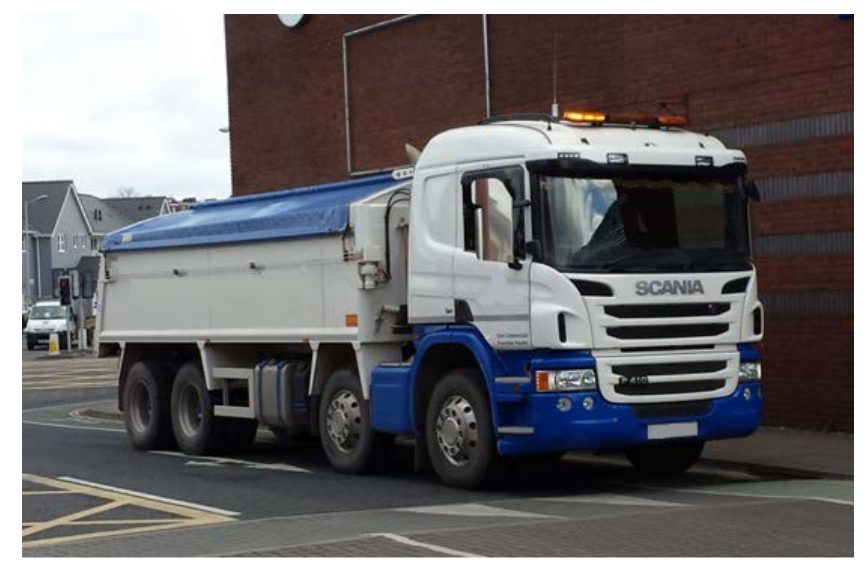

(a)

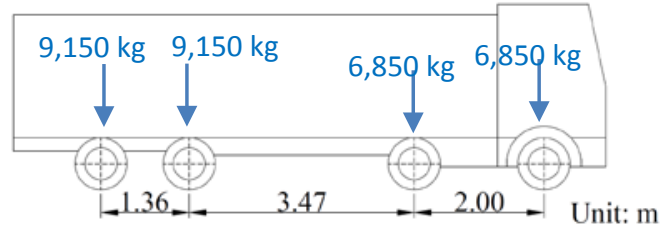

(b)

556 Fig. 14, Truck used in the load test, (a) photo of the truck, (b) axle spacing.

557

558

559

560

561

562

563

564

565

566

567

568

569

570

571

\subsection{Results}

Fig. 15 shows the Imetrum displacement data and the corresponding QA acceleration data for the bridge test with acceleration $y$-axis on the left and the displacement $y$-axis on the right. Within the 16 minutes (960 seconds) test duration the truck crossed the bridge six times as indicated in the six corresponding clear peaks in Fig. 15.

The load corresponding to each peak can be checked using camera 2 video, for example for the first deflection peak in Fig. 15 at approximately 230 seconds, Fig. 16 shows camera 2 view at 229 seconds just before the truck reaches mid-span (if the image from 230 seconds is used it is more difficult to identify the truck). The corresponding acceleration pulses do not stand out in Fig. 15, in fact acceleration pulses occur when any kind of vehicle enters the bridge passing over the irregular surface near the support. To identify the acceleration pulses corresponding to truck loading and integrate the correct part of the signal it is necessary to identify the crossing time in the video. With the approximate time of the truck crossing event known, zooming on the acceleration signals allows the corresponding acceleration segment to be identified, as shown for swipe 1 and swipe 2 in Figs. $17(a)$ and (d) respectively. 


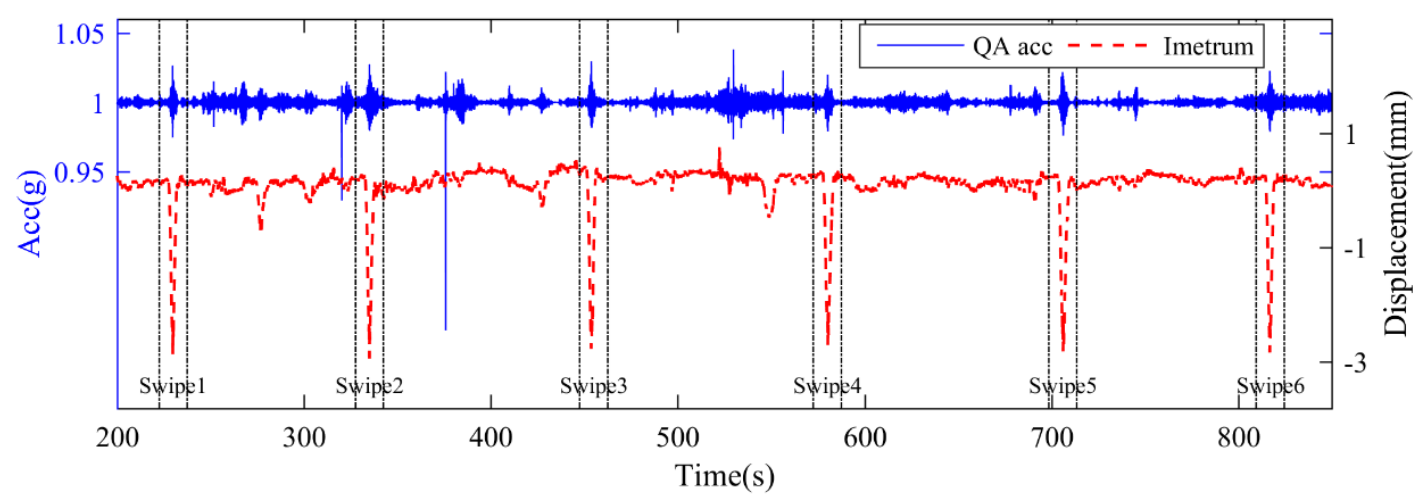

572

Fig. 15, Imetrum displacement data and QA acceleration data for the bridge test.

574

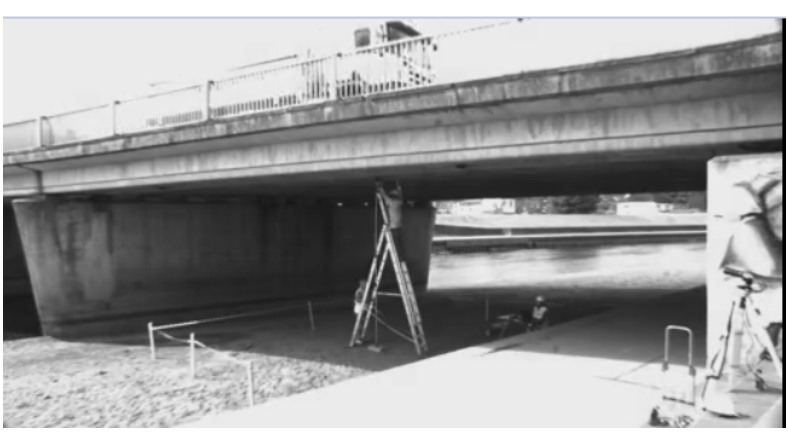

576 Fig. 16, Loading on bridge at 229 seconds, i.e. truck crossing over bridge during swipe 1.

577 Fig. 17 shows the integration procedure applied to swipe 1 and swipe 2 of the field test and is 578 analogous to Fig. 11. Fig. 17(a-c) for swipe 1, show the de-trended acceleration signal, the calculated 579 velocity, and the calculated displacement respectively. Camera 2 shows the truck entering the bridge 580 at 228 seconds and leaving at 232 seconds, which is indicated by dashed and solid lines, respectively, 581 in the figure. Fig. 17(c) shows the calculated displacement before the truck arrives and after it leaves 582 to be very close to zero, i.e. the pre-load and post-load parts of the signal appear to be correct, 583 which (based on results observed in the laboratory) indicates that the peak displacement of $2.8 \mathrm{~mm}$ 584 for the forced part of the signal is likely to be reliable. For swipe 2 with truck entering the bridge at 585333 seconds and leaving at approximately 337 seconds pre-load displacement is correct (i.e. $0 \mathrm{~mm}$ ), 586 while post-load part of the signal reads approximately $-0.35 \mathrm{~mm}$, which quantifies the likely error in the forced part of the calculated displacement signal. 


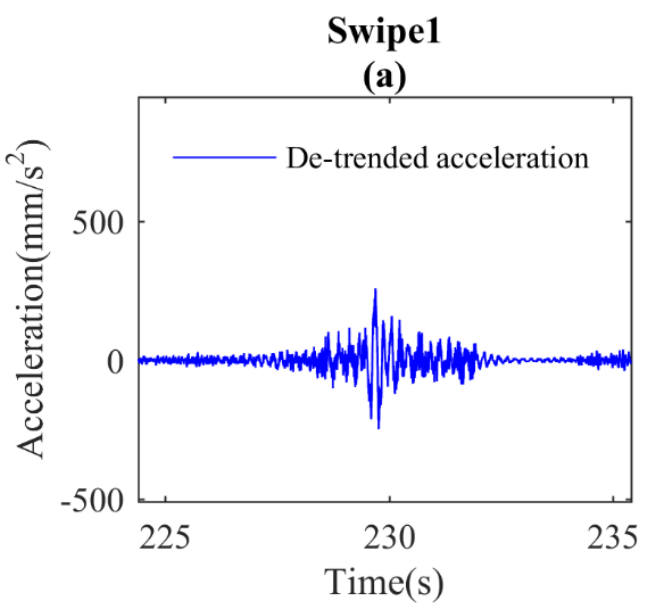

(b)

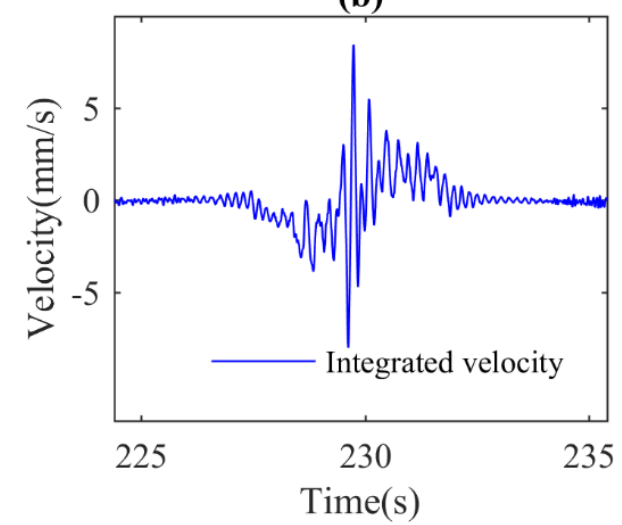

(c)

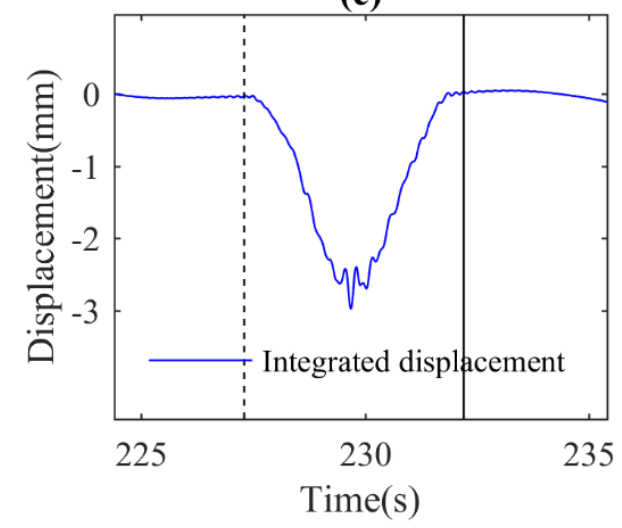

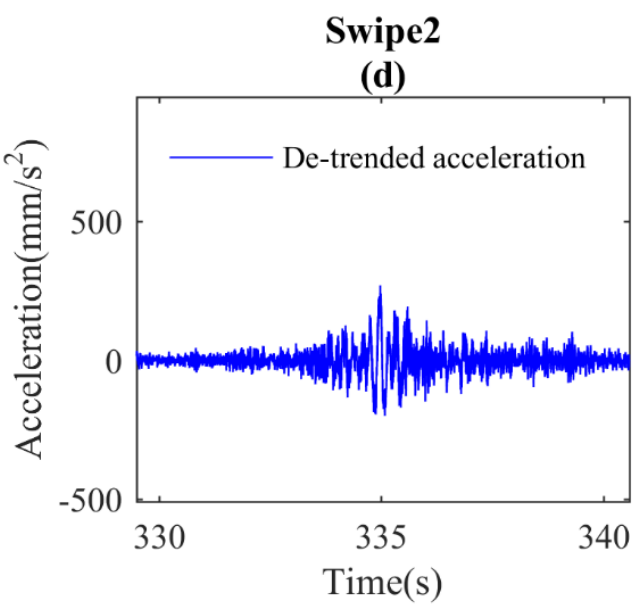

(e)

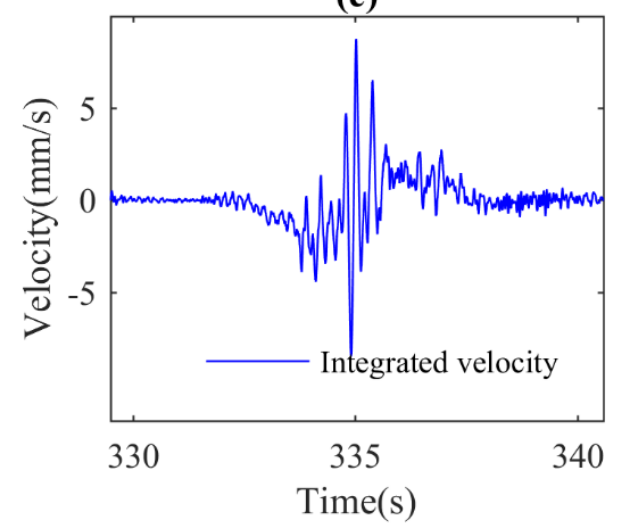

(f)

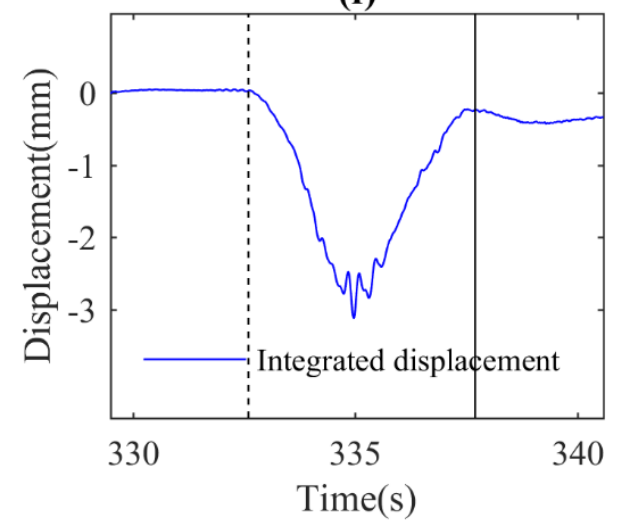

589 Fig 17, Calculating displacement from acceleration for swipe 1 (left column) and swipe 2 (right 590 column) of bridge test; (a) swipe 1 acceleration, (b) swipe 1 velocity calculated following integration 591 of acceleration signal, (c) swipe 1 displacement calculated from integration of velocity signal, (d) 592 swipe 2 acceleration, (e) swipe 2 velocity calculated following integration of acceleration signal, (f) swipe 2 displacement calculated following integration of velocity signal.

Fig. 18 shows the calculated (QA) and measured (Imetrum \& LVDT) displacement for all six swipes and broadly speaking there is good agreement between the three signals. As with the integration 
597 process for the analog bridge, initial displacement and velocity at the start of the swipe are assumed

598 to be zero, an assumption validated in Fig. 18. This would not hold true in the case of another heavy

599 vehicle leaving the bridge, but this would be spotted in the video.

600 In Fig. 18(b) the LVDT data shows an anomaly at approximately 339 seconds resulting from the 601 difficulty experienced in keeping the LVDT setup stable during the test. Occasionally the strong wind 602 experienced during the test would move the tip of the LVDT laterally out of the dimple recess before 603 having to be reset manually. This is the likely cause for the displacement anomaly at 339 seconds 604 and most likely for the anomaly at 852 seconds in Fig. 18(d).

605 The observation from the field test, supported by the analog bridge test is that 'close to zero' pre606 load and post-load displacement indicate a credible displacement time history. Since the quality 607 assurance requires short periods before and after the truck passes with no other heavy vehicles 608 (trucks) present means that the proposed procedure would not be suitable during periods of busy 609 traffic. It is also limited to relatively short bridges because of more frequent truck passing and the 610 increasing unreliability of double integration. However in the 'small hours' around 4AM it is usual to 611 find periods when even the busiest bridge may be empty long enough for a measurement to be 612 taken. 
(a) Swipe 1

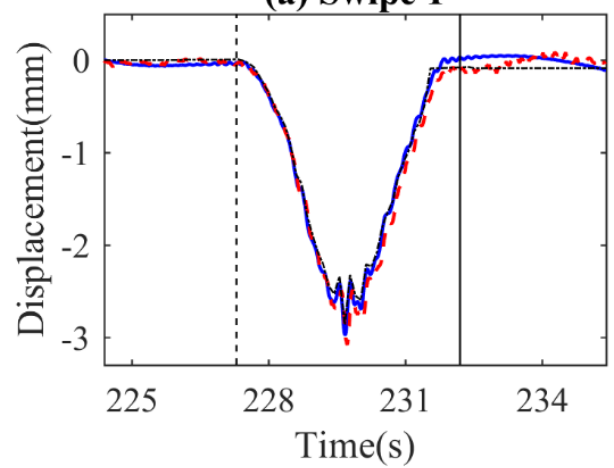

(c) Swipe 3

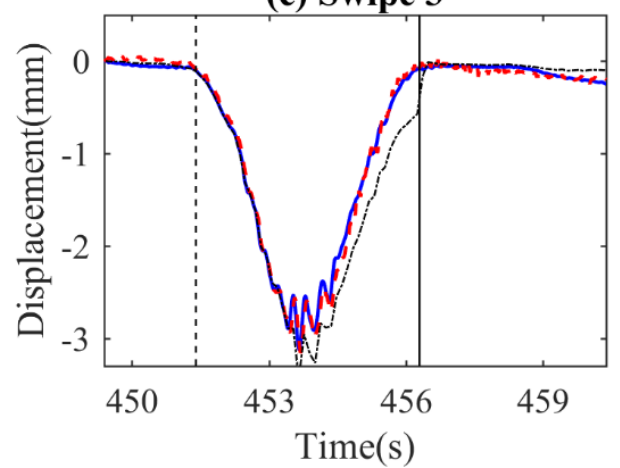

(e) Swipe 5

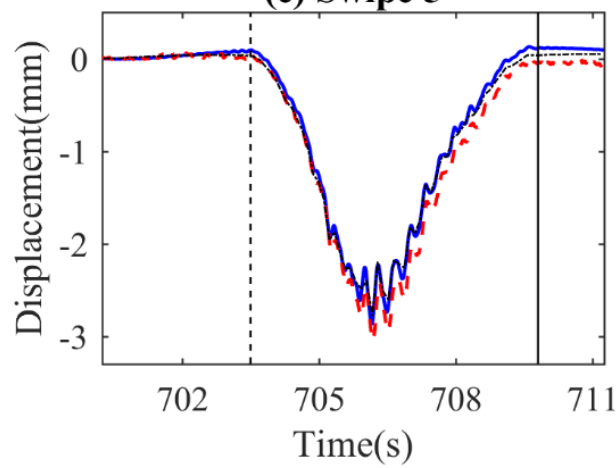

(b) Swipe 2

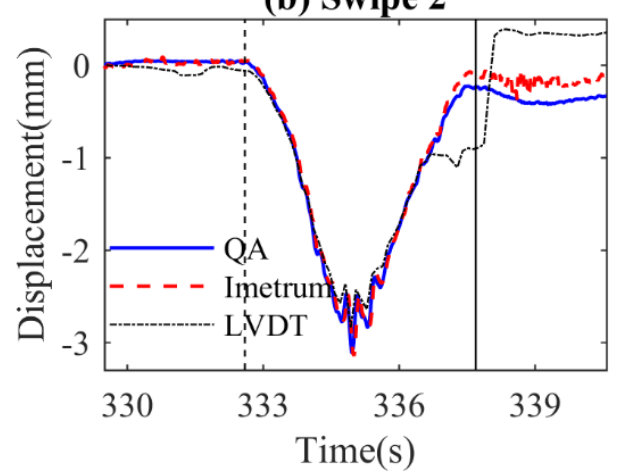

(d) Swipe 4

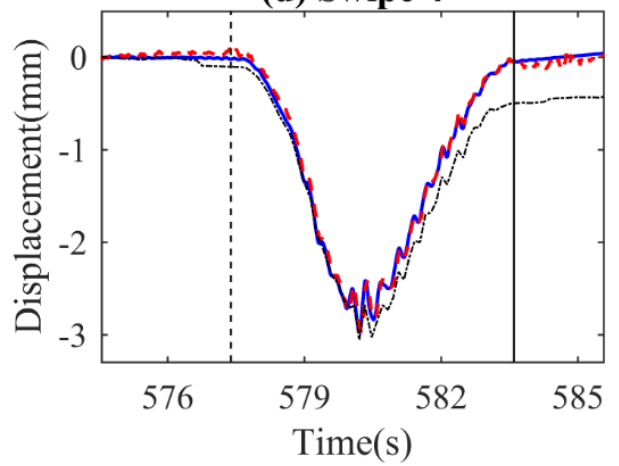

(f) Swipe 6

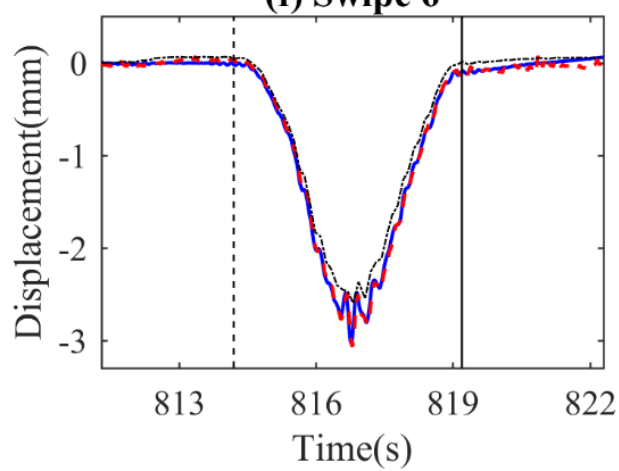

619

620 Fig 18, Displacement observed for the three measurement systems for swipes 1-6 (a) swipe 1, (b)

621 swipe 2 (c) swipe 3, (d) swipe 4, (e) swipe 5, (f) swipe 6.

622

623

624

625

626 


\subsection{Conclusions}

628 This paper presents a method for carrying out a low cost minimally invasive bridge load test by

629 calculating bridge displacement to a moving load from the bridge acceleration response. The

630 performance of the method is examined in both laboratory and field conditions and accurate results

631 are observed in both tests. The paper presents two novel contributions to existing work in this area.

632 Firstly the paper demonstrates the importance of low frequency accelerometer noise. Selection of

633 accelerometer was done by comparing performance of a range of sensors. A simple numerical model

634 demonstrates how the quasi-static component of bridge displacement response to a moving load

635 appears in the bridge acceleration signal in time and frequency domain.

636 Second, the proposed method provides an in-built quality control check on the calculated signal.

637 Methods presented in the literature to calculate bridge displacement from acceleration have

638 demonstrated their accuracy in one off experiments by comparing the 'calculated' displacement to

639 the displacement measured directly using e.g. LVDT. However without such a reference it has been

640 impossible to estimate accuracy in the calculated displacement. The approach proposed here uses

641 video footage synchronised with acceleration signals to estimate the magnitude of the likely errors

642 in the calculated displacement signal.

643 The paper presents an extensive experimental study in both laboratory and field conditions with

644 displacement measured directly by LVDT and an optical tracking system. The two direct

645 measurement systems validated each other and provided error estimates for the calculated

646 displacements. The laboratory trial aiming to reproduce the field trial in terms of truck-passing

647 duration and deflection with total control of loading was very effective and provided the means to

648 judge the reliability of the field test data.

649 While the errors observed in the calculated displacement in this paper are pleasingly small, it is 650 important to understand that the results relate to a specific set of circumstances. Namely; (i) short

651 duration time intervals, (ii) small amplitude bridge accelerations and (iii) small amplitude

652 displacements. The significance of each of these factors in obtaining an accurate 'calculated

653 displacement' has been well demonstrated/explained by others [21], [22]. Therefore an in depth

654 discussion on this is not repeated here, except to point out that if that approach used in this paper

655 was applied to circumstances where one or more of (i), (ii) or (iii) were not true, (e.g. suspension

656 bridge with a long duration acceleration signal, or an earthquake event with high accelerations) large

657 errors could result.

658 Traditionally a bridge load test is a complicated exercise requiring specialist equipment and

659 operators along with road closure. This study shows that a simple measurement using a high quality

660 accelerometer and a video camera, performed in the middle of the night when prevailing traffic

661 volumes are relatively low could provide a reliable deflection estimate for a known load. However, it

662 is important to note that the authors are not claiming that the approach presented in the paper is

663 necessarily the best or most practical approach for logistically feasible load testing. The improving

664 accuracy of GPS to measure vertical displacement and the emergence of camera based displacement

665 monitoring means that there a number of options to measure bridge displacement during a load

666 test. Ultimately the displacement tracking method used during a load test will likely depend on the

667 equipment available, the logistics of the site and the expertise/experience of the test crew. 
Therefore the aim of this paper is not to promote one method over another, instead the intention is to describe the author's experiences of integrating bridge acceleration to calculate displacement and to report the potential benefits and limitations of the approach.

\section{Acknowledgements}

The research leading to these results has received funding from the People Programme (Marie Curie Actions) of the European Union's Seventh Framework Programme (FP7/2007-2013) under grant agreement $n^{\circ} 330195$. The authors would also like to acknowledge the Bridge Section of The Engineering Design Group of Devon County Council led by Kevin Dentith BSc, CEng, FICE, for their support and assistance with this work. Finally the authors would like to thank the three anonymous reviewers for their constructive comments.

\section{References}

[1] J. McConnell, M. Chajes, and K. Michaud, "Field Testing of a Decommissioned Skewed Steel IGirder Bridge: Analysis of System Effects," J. Struct. Eng., vol. 141, no. 1, p. D4014010, Jul. 2014.

[2] Standards_for_highways, Design Manual for Roads and Bridges (DMRB) Volume 3 Section 4 Part 3 (BD 21/01) Highway structures: Inspection and maintenance assessment. The assessment of highway bridges and structures. 2001.

[3] Standards_for_highways, Design Manual for Roads and Bridges (DMRB) Volume 3 Section 4 Part 8 (BA 54/94) Highway structures: Inspection and maintenance assessment. Load testing for bridge assessment. 1994.

[4] J. M. W. Brownjohn, K.-Y. Koo, A. Scullion, and D. List, "Operational deformations in long-span bridges," tructure Infrastruct. Eng., vol. 11, pp. 556-574, 2015.

[5] H. Le, S. Cho, R. E. Kim, F. Moreu, S. Scola, H. Jo, J. M. LaFave, J. Li, B. F. Spencer, and A. Kimmle, "Dynamic Assessment of Timber Railroad Bridges Using Displacements," J. Bridg. Eng., vol. 20, no. 10, Oct. 2015.

[6] S. C. Stiros and P. A. Psimoulis, "Response of a historical short-span railway bridge to passing trains: 3-D deflections and dominant frequencies derived from Robotic Total Station (RTS) measurements," Eng. Struct., vol. 45, pp. 362-371, 2012.

[7] K. Wong, K. Man, and W. Chan, "Monitoring Hong Kong's bridges - Real-time Kinematic spans the gap, GPS World," 2001. [Online]. Available: http://grupos.unican.es/gidai/web/asignaturas/Cl/Bridges.pdf. [Accessed: 12-Apr-2016].

[8] W. Roberts, G, A. H. Dodson, and V. Ashkenazi, "Twist and deflect, monitoring motion of the Humber Suspension Bridge," GPS World, pp. 24-34, 1999.

[9] F. Moschas and S. C. Stiros, "Three-dimensional dynamic deflections and natural frequencies of a stiff footbridge based on measurements of collocated sensors," Struct. Control Heal. Monit., vol. 21, no. 1, pp. 23-42, Jan. 2014.

[10] Imetrum, "Imetrum road bridges." [Online]. Available: http://www.imetrum.com/roadbridges/.

[11] T. Khuc and F. N. Catbas, "Completely contactless structural health monitoring of real-life 
structures using cameras and computer vision," Struct. Control Heal. Monit., p. 17, 2016.

[12] T. Khuc and F. N. Catbas, "Computer vision-based displacement and vibration monitoring without using physical target on structures," Struct. Infrastruct. Eng., pp. 1-12, Apr. 2016.

[13] D. Ribeiro, R. Calçada, J. Ferreira, and T. Martins, "Non-contact measurement of the dynamic displacement of railway bridges using an advanced video-based system," Eng. Struct., vol. 75, pp. 164-180, Sep. 2014.

[14] B. C. Faulkner, F. W. Barton, T. T. Baber, and W. T. McKeel, "Determination of Bridge Response Using Acceleration Data," 1996.

[15] P. Paultre, J. Proulx, and M. Talbot, "Dynamic Testing Procedures for Highway Bridges Using Traffic Loads," J. Struct. Eng., vol. 121, no. 2, pp. 362-376, 1995.

[16] K. T. Park, S. H. Kim, H. S. Park, and K. W. Lee, "The determination of bridge displacement using measured acceleration," Eng. Struct., vol. 27, no. 3, pp. 371-378, 2005.

[17] M. Gindy, H. Nassif, and J. Velde, "Bridge Displacement Estimates from Measured Acceleration Records," Transp. Res. Rec. J. Transp. Res. Board, vol. 2028, no. 2028, pp. 136145, Dec. 2007.

[18] P. Kropp, "Experimental Study of the Dynamic Response of Highway Bridges : Interim Report," 1977.

[19] M. Gindy, R. Vaccaro, H. Nassif, and J. Velde, "A State-Space Approach for Deriving Bridge Displacement from Acceleration," Comput. Civ. Infrastruct. Eng., vol. 23, no. 4, pp. 281-290, May 2008.

[20] Y. K. Thong, M. S. Woolfson, J. A. Crowe, B. R. Hayes-Gill, and D. A. Jones, "Numerical double integration of acceleration measurements in noise," Meas. J. Int. Meas. Confed., vol. 36, no. 1, pp. 73-92, 2004.

[21] S. C. Stiros, "Errors in velocities and displacements deduced from accelerographs: An approach based on the theory of error propagation," Soil Dyn. Earthq. Eng., vol. 28, no. 5, pp. 415-420, 2008.

[22] F. Moschas, D. Mouzoulas, and S. Stiros, "Phase errors in accelerometer arrays: An analysis based on collocated sensors and FEM," Soil Dyn. Earthq. Eng., vol. 78, pp. 32-45, 2015.

[23] J. M. W. Brownjohn and A. Pavic, "Vibration control of ultra-sensitive facilities," Struct. Build., vol. 159, no. SB5, pp. 295-306, 2006.

[24] L. Heng, Y. Yunsheng, Z. Shuiming, C. Yongjian, and L. Dongning, "Baseline correction for digital strong-motion records by using the pre-event portion," Geod. Geodyn., vol. 2, no. 1, pp. 43-46, Feb. 2011.

[25] G. M. Graizer, "Determination of the true ground displacement by using strong motion records," Izv. Phys. Solid Earth, vol. 15, pp. 875-886, 1979.

[26] L. Zhu, "Recovering permanent displacements from seismic records of the June 9, 1994 Bolivia deep earthquake," Geophys. Res. Lett., vol. 30, no. 14, p. n/a-n/a, Jul. 2003.

[27] D. M. Boore and S. Akkar, "Effect of causal and acausal filters on elastic and inelastic response spectra," Earthq. Eng. Struct. Dyn., vol. 32, no. 11, pp. 1729-1748, Sep. 2003. 
761

2

[28] W. Graves, Robert, "Processing Issues for Near Source Strong Motion Recordings. Presented," in Workshop on Strong-Motion Recording Processing, Consortium of Organizations for StrongMotion Observation Systems, 2004, pp. 193-201.

[29] D. M. Boore, "Effect of Baseline Corrections on Displacements and Response Spectra for Several Recordings of the 1999 Chi-Chi, Taiwan, Earthquake," Bull. Seismol. Soc. Am., vol. 91, no. 5, pp. 1199-1211, Oct. 2001.

[30] W. D. Iwan, M. A. Moser, and P. Chia-Yen, "SOME OBSERVATIONS ON STRONG-MOTION EARTHQUAKE MEASUREMENT USING A DIGITAL ACCELEROGRAPH.," Bull. Seismol. Soc. Am., vol. 75, no. 5, pp. 1225-1246, 1985.

[31] A. González and D. Hester, "An investigation into the acceleration response of a damaged beam-type structure to a moving force," J. Sound Vib., vol. 332, no. 13, pp. 3201-3217, Jun. 2013.

[32] P. Psimoulis, N. Houile, M. Meindl, and M. Rothacher, "Consistency of PPP GPS and strongmotion records: case study of Mw9.0 Tohoku-Oki 2011 earthquake," Smart Struct. Syst., vol. 16, no. 2, pp. 347-346, 2015. 